\title{
The Ingram conjecture
}

\author{
MARCY BARGE \\ HENK BRUIN \\ SONJA ŠTIMAC
}

\begin{abstract}
We prove the Ingram conjecture, ie we show that the inverse limit spaces of tent maps with different slopes in the interval $[1,2]$ are nonhomeomorphic. Based on the structure obtained from the proof, we also show that every self-homeomorphism of the inverse limit space of a tent map is pseudo-isotopic, on the core, to some power of the shift homeomorphism.
\end{abstract}

54H20; 37B45, 37E05

\section{Introduction}

Apart from their interest within continuum theory, inverse limit spaces play a key role in the description of uniformly hyperbolic attractors (see Williams [35; 36]), global "Hénon-like" strange attractors (Barge and Holte [6]) and the structure emerging from homoclinic tangencies in dynamical systems (Barge and Diamond [5]). They find further use in the area of (substitution) tiling spaces (Anderson and Putnam [1]) which, in some cases, are covering spaces of the type of inverse limit spaces with which we are concerned with in this paper; namely, those with a single tent map $T_{s}:[0,1] \rightarrow[0,1]$, $x \mapsto \min \{s x, s(1-x)\}$ as bonding map. Such inverse limit spaces can be embedded in the plane as global attractors of homeomorphisms (see Bruin [13], Misiurewicz [26] and Szczechla [33]) and immersed in the plane as global attractors of skew product maps (Hofbauer, Raith and Simon [20]).

Inverse limit spaces are notoriously difficult to classify. In this paper, we solve in the affirmative the classification problem known as the Ingram conjecture:

Theorem 1.1 (Ingram conjecture) If $1 \leq s<s^{\prime} \leq 2$, then the corresponding inverse limit spaces $\lim _{\longleftarrow}\left([0,1], T_{s}\right)$ and $\lim _{\longleftarrow}\left([0,1], T_{s^{\prime}}\right)$ are nonhomeomorphic.

This is the main outstanding conjecture regarding dynamics on continua, dating back to at least the early nineties. In [21, page 257] in 1995, Ingram writes: 
The [...] question was asked of the author by Stu Baldwin at the [...] summer meeting of the AMS at Orono, Maine, in 1991. ... There is a related question which the author has considered to be of interest for several years. He posed it at a problem session at the 1992 Spring Topology Conference in Charlotte for the special case (that the critical point has period) $n=5$.

It is clear that if two interval maps are topologically conjugate, then their inverse limit spaces are homeomorphic. Thus it may be more natural to ask the question for the "fuller" logistic family $f_{a}(x)=a x(1-x), a \in[0,4]$, which includes (infinitely) renormalizable maps (see Definition 6.1). It is well-known (Milnor and Thurston [25]) that each logistic map with positive topological entropy is semiconjugate to a tent map $T_{s}$ with $\log s=h_{\text {top }}\left(f_{a}\right)$, and these semiconjugacies collapse (pre)periodic intervals to points. The effect of renormalization on the structure of the inverse limit space is well-understood; see Barge and Diamond [4]: it produces proper subcontinua that are periodic under the shift homeomorphism and homeomorphic with the inverse limit space of the renormalized map. The solution of the Ingram conjecture then leads to an analogous result for logistic maps.

As the parameter $a$ for the logistic map $f_{a}$ is increased, a new periodic point of period $k$ appears when the graph of $f_{a}^{k}$ is tangent with the diagonal. If such a tangency happens at parameter value $a_{*}$ and location $(x, x)$ then there is an $\epsilon>0$ and a pair of continuous curves $a \mapsto x_{-}(a), a \mapsto x_{+}(a)$ of $k$-periodic points with $x_{-}\left(a_{*}\right)=x=x_{+}\left(a_{*}\right)$ so that $x_{-}(a)$ is repelling and $x_{+}(a)$ is attracting under $f_{a}^{k}$, for $a_{*}<a \leq a_{*}+\epsilon$. This is called a saddle-node bifurcation and $\left\{x_{-}(a), x_{+}(a)\right\}$ is called a saddle-node pair. For each $a$, let $\sim_{s n}$ be the equivalence relation that identifies saddle-node pairs and let $\Omega\left(f_{a}\right)$ be the nonwandering set of $f_{a}$ (see Definition 6.2). The reduced nonwandering set of $f_{a}$ is $\Omega\left(f_{a}\right) / \sim_{s n}$.

Theorem 1.2 In the parameter range $(1,4]$, two logistic maps have homeomorphic inverse limit spaces if and only if they are conjugate on their reduced nonwandering sets.

There have been several partial results to the Ingram conjecture, eg Barge and Diamond [3], who solved the period $n=5$ case, and Swanson and Volkmer [32] and Bruin [14]. The Ingram conjecture was shown to hold when the critical point is periodic by Kailhofer [22] (see also Block, Jakimovik, Keesling and Kailhofer [8]), or has finite orbit by Štimac [31]. More recently, the case where the critical point is nonrecurrent was solved by Raines and Štimac [29]. Further results that classify certain features of inverse limit spaces of tent maps with nonperiodic recurrent critical orbits were obtained by Barge, Brucks and Diamond [11], Bruin [15] and Raines [28]. 
Our solution to the Ingram conjecture gives more information about the set of selfhomeomorphisms on $\lim \left([0,1], T_{s}\right)$ : we show that any such homeomorphism behaves like an iterate of the shift homeomorphism $\sigma$.

The critical point $\frac{1}{2}$ of $T_{s}$ is denoted by $c$, and we write $c_{i}=T_{s}^{i}(c)$. Although $T_{s}$ is defined on $[0,1]$, there is a forward invariant interval $\left[c_{2}, c_{1}\right]=[s(1-s / 2), s / 2]$, called the core, on which $T_{S}$ is surjective. We call $\lim \left(\left[c_{2}, c_{1}\right], T_{S}\right)$ the core of the inverse limit space. The space $\lim \left([0,1], T_{s}\right)$ is the union of the core of the inverse limit and a ray $\mathfrak{C}$ converging onto $\overleftarrow{i t}$.

Recall that the composant of $x \in X$ is defined as the union of all proper subcontinua of $X$ containing $x$. For $1<s<2, \lim \left([0,1], T_{s}\right)$ has only three composants: the entire inverse limit space, $\mathfrak{C}$, and $\left.\lim \overleftarrow{([0}, 1], T_{s}\right) \backslash\{(\ldots, 0,0,0)\}$. But for $s>\sqrt{2}$, $\lim \left(\left[c_{2}, c_{1}\right], T_{s}\right)$ is indecomposable $\overleftarrow{\text { and }}$ hence has uncountably many pairwise disjoint composants, each of which is dense. If $s>\sqrt{2}$ and the orbit of $c$ is finite, the composants of $\lim \left(\left[c_{2}, c_{1}\right], T_{s}\right)$ are the same as the arc-components. Otherwise, the

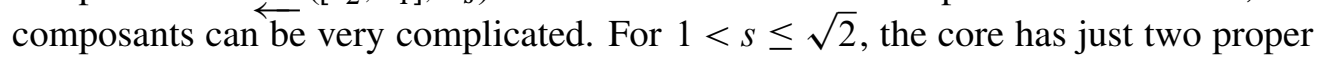
composants that overlap in a single arc-component.

Theorem 1.3 Given $s \in[1,2]$, for every homeomorphism $h$ : $\lim \left([0,1], T_{s}\right) \circlearrowleft$, there is an $R \in \mathbb{Z}$ such that $h$, restricted to the core $\lim _{\longleftarrow}\left(\left[c_{2}, c_{1}\right], T_{s}\right)$, is pseudo-isotopic to $\sigma^{R}$, ie it permutes the composants of the core of the inverse limit in the same way as $\sigma^{R}$.

The zero-composant $\mathfrak{C}$ of $\lim \left([0,1], T_{S}\right)$ containing the endpoint $\alpha:=(\ldots, 0,0,0)$ is important in our proof of the Ingram conjecture; the "core" version of the Ingram conjecture is still outstanding. Our proof relies on the properties of so-called linksymmetric arcs in the composant $\mathfrak{C}$. Inverse limit spaces are chainable, and with regard to natural chains, a homeomorphism $h: \lim \left([0,1], T_{s^{\prime}}\right) \rightarrow \underset{\leftarrow}{\lim }\left([0,1], T_{s}\right)$ maps link-symmetric arcs to link-symmetric arcs. From this we derive $\overleftarrow{\leftarrow}$ at maximal linksymmetric arcs in $\lim _{\longleftarrow}\left([0,1], T_{s^{\prime}}\right)$ centered at so-called salient points $s_{i}^{\prime}$ map to linksymmetric arcs centered at salient points $s_{i+M} \in \underset{\lim }{\longleftarrow}\left([0,1], T_{s}\right)$ for some $M \in \mathbb{Z}$ and all sufficiently large $i \in \mathbb{N}$.

This implies that $h$ maps so-called $q$-points close to $p$-points, while "translating" their levels by a fixed number $M$. This shows that $h$ effectively fixes the folding pattern of the zero-composant, with the Ingram conjecture as an easy consequence. Additional arguments show that every self-homeomorphism of $\lim \left([0,1], T_{S}\right)$, when restricted to the core, is pseudo-isotopic to a power $\sigma^{R}$ of the shift for some $R \in \mathbb{Z}$.

We give the basic definitions in the next section. In Section 3 we investigate the lengths of maximal link-symmetric arcs, leading in Section 4 to the proof that a homeomorphism 
between two unimodal inverse limit spaces induces a shift of indices of salient points, and more generally, acts as a shift on the levels of $q$-points and $p$-points. This leads to the proof of the Ingram conjecture. In Section 5, we prove the results on pseudo-isotopy. Section 6, finally, is devoted to the proof of Theorem 1.2 on logistic maps.

\section{Definitions}

Let $\mathbb{N}:=\{1,2, \ldots\}$ and $\mathbb{N}_{0}:=\{0,1,2, \ldots\}$. Let

$$
T_{s}:[0,1] \rightarrow[0, s / 2], \quad T_{s}(x)=\min \{s x, s(1-x)\},
$$

be the tent map with slope $s \in[1,2]$ and critical point $c=\frac{1}{2}$. Write $c_{i}=c_{i}(s):=T_{s}^{i}(c)$, so in particular $c_{1}=\frac{s}{2}$ and $c_{2}=s\left(1-\frac{s}{2}\right)$.

The inverse limit space $\lim _{\longleftarrow}\left([0,1], T_{S}\right)$ is the collection of backward orbits

$$
\left\{x=\left(\ldots, x_{-2}, x_{-1}, x_{0}\right): T_{s}\left(x_{i-1}\right)=x_{i} \in[0, s / 2] \text { for all } i \leq 0\right\},
$$

equipped with metric $d(x, y)=\sum_{n \leq 0} 2^{n}\left|x_{n}-y_{n}\right|$ and induced (or shift) homeomorphism $\sigma=\sigma_{s}$ given by

$$
\sigma\left(\ldots, x_{-2}, x_{-1}, x_{0}\right)=\left(\ldots, x_{-2}, x_{-1}, x_{0}, T_{s}\left(x_{0}\right)\right) .
$$

Let $\pi_{p}: \lim \left([0,1], T_{s}\right) \rightarrow[0,1], \pi_{p}(x)=x_{-p}$, be the $p$-th projection map. Since $T_{s}$ fixes $0, \overleftarrow{\lim }\left([0,1], T_{S}\right)$ contains the endpoint $\alpha:=(\ldots, 0,0,0)$. The proper composant of $\lim \left([\overleftarrow{0,1}], T_{s}\right)$ containing this point is denoted by $\mathfrak{C}$; it is a ray converging from $\alpha$

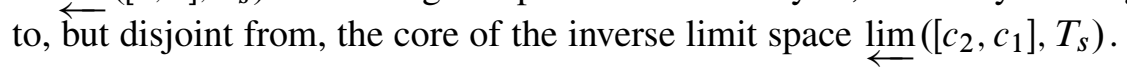

Frequently, the Ingram conjecture is posed for slopes $s, s^{\prime} \in[\sqrt{2}, 2]$ only, because for $0<s \leq \sqrt{2}, \lim \left(\left[c_{2}, c_{1}\right], T_{s}\right)$ is decomposable. Since $\lim _{\longleftarrow}\left([0,1], T_{s}\right)$ is a single point for $s \in(0,1)$ and an arc for $s=1$, we will always assume that all slopes $s$ are greater than 1 . The next two lemmas show how to reduce the case $s \in(1, \sqrt{2}]$ to $s \in(\sqrt{2}, 2]$.

Lemma 2.1 For $2^{1 / 2^{n+1}} \leq s \leq 2^{1 / 2^{n}}, n \in \mathbb{N}$, the core of the inverse limit space $\underset{\lim }{\longleftarrow}\left(\left[c_{2}, c_{1}\right], T_{s}\right)$ is homeomorphic with two copies of $\lim _{\longleftarrow}\left([0,1], T_{s^{2}}\right)$ joined at their $\overleftarrow{\text { endpoints. }}$

Proof For this range of $s, T_{s}\left(\left[c_{2}, p\right]\right)=\left[p, c_{1}\right]$ and $\left.T_{s}\left(\left[p, c_{1}\right]\right)=\left[c_{2}, p\right]\right)$, where $p:=s /(s+1)$ is the positive fixed point of $T_{s}$. It follows that $\lim \left(\left[c_{2}, c_{1}\right], T_{s}\right)$ is homeomorphic with two copies of $\lim \left(\left[p, c_{1}\right], T_{s}^{2}\right)$ joined at the endpoint $(\ldots, p, p, p)$. Direct calculation shows that, if $L \overleftarrow{\text { is }}$ the orientation preserving affine homeomorphism from $\left[p, c_{1}\right]$ onto $\left[0, c_{1}\left(s^{2}\right)\right]$, then $L \circ T_{s}^{2} \circ L^{-1}=T_{s^{2}}$ on $\left[0, c_{1}\left(s^{2}\right)\right]$ and hence $\underset{\lim }{\longleftarrow}\left(\left[p, c_{1}\right], T_{s}^{2}\right)$ is homeomorphic with $\underset{\longleftarrow}{\lim }\left([0,1], T_{s^{2}}\right)$. 
Lemma 2.2 Suppose that $2^{1 / 2^{n}}<s \leq 2^{1 / 2^{n-1}}$ and $2^{1 / 2^{n^{\prime}}}<s^{\prime} \leq 2^{1 / 2^{n^{\prime}-1}}, n, n^{\prime} \in \mathbb{N}$, and suppose that $\lim \left([0,1], T_{s}\right)$ is homeomorphic with $\lim _{\longleftarrow}\left([0,1], T_{s^{\prime}}\right)$. Then $n=n^{\prime}$ and assuming that the Ingram conjecture holds for slopes strictly greater than $\sqrt{2}$, then $\underset{\lim }{\longleftarrow}\left([0,1], T_{s^{2^{n-1}}}\right)$ is also homeomorphic with $\underset{\longleftarrow}{\lim }\left([0,1], T_{\left(s^{\prime}\right)^{2 n-1}}\right)$.

Proof For $2^{1 / 2}<s<2, \lim \left([0,1], T_{s}\right)$ consists of a ray $\mathfrak{C}$ winding onto an indecomposable continuum, namely $\lim \left(\left[c_{2}, c_{1}\right], T_{s}\right)$. It follows from Lemma 2.1 that for $2^{1 / 2^{n}}<s<2^{1 / 2^{n-1}}, \lim \left([0,1], T_{s}\right)$ consists of a ray winding onto a pair of rays, each winding onto a pair of rays, ..., each winding onto a pair of rays, each of which winds onto an indecomposable continuum. There are $2^{n-1}$ of these indecomposable continua, each homeomorphic with the core of the inverse limit space $\lim \left([0,1], T_{s^{2^{n-1}}}\right)$. Hence if $\lim \left([0,1], T_{s}\right)$ is homeomorphic with $\underset{\leftarrow}{\lim }\left([0,1], T_{s^{\prime}}\right)$,

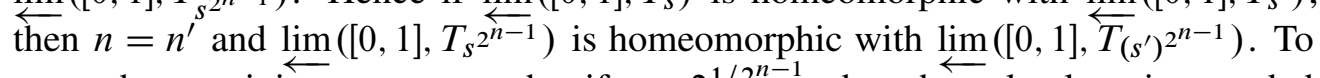
cover the remaining cases, note that if $s=2^{1 / 2^{n-1}}$, then the only alteration needed in the above description of $\lim \left([0,1], T_{S}\right)$ is that at the penultimate level, instead of a pair of rays winding onto a pair of indecomposable subcontinua, we just have two indecomposable subcontinua (each homeomorphic with $\lim _{\longleftarrow}\left([0,1], T_{2}\right)$ ) joined at their common endpoint. It is then clear that if $\lim _{\longleftarrow}\left([0,1], T_{s^{\prime}}\right)$ is homeomorphic with $\underset{\lim }{\longleftarrow}\left([0,1], T_{s}\right)$, then $s^{\prime}=s$.

Definition 2.3 The arc-length or $\bar{d}$ metric on $\mathfrak{C}$ is defined as

$$
\bar{d}(x, y)=s^{p}\left|x_{-p}-y_{-p}\right|
$$

for each $p$ so that $\pi_{p}:[x, y] \rightarrow[0,1]$ is injective.

If $x, y \in \mathfrak{C}$, then we denote by $[x, y]$ the arc between $x$ and $y$, and by $(x, y)$ the interior of the arc $[x, y]$. We write $x \preceq y$ if $x \in[\alpha, y]$, ie $\bar{d}(\alpha, x) \leq \bar{d}(\alpha, y)$.

Definition 2.4 A continuum is chainable if for every $\varepsilon>0$, there is a cover $\left\{\ell^{1}, \ldots, \ell^{n}\right\}$ of open sets (called links) of diameter $<\varepsilon$ such that $\ell^{i} \cap \ell^{j} \neq \varnothing$ if and only if $|i-j| \leq 1$. Such a cover is called a chain. Clearly the interval $[0, s / 2]$ is chainable. We call a sequence $\mathcal{C}_{p}, p \in \mathbb{N}_{0}$, a natural chaining, and each $\mathcal{C}_{p}$ a natural chain, of $\underset{\lim }{\longleftarrow}\left([0,1], T_{S}\right)$, if

(1) there is a chain $\left\{I_{p}^{1}, I_{p}^{2}, \ldots, I_{p}^{n}\right\}$ of $[0, s / 2]$ such that $\ell_{p}^{j}:=\pi_{p}^{-1}\left(I_{p}^{j}\right)$ are links of $\mathcal{C}_{p}$

(2) each point $x \in \bigcup_{i=0}^{p} T_{s}^{-i}(c)$ is a boundary point of some link $I_{p}^{j}$;

(3) for each $i$ there is $j$ such that $T_{s}\left(I_{p+1}^{i}\right) \subset I_{p}^{j}$. 
Let us define width $\left(\mathcal{C}_{p}\right):=\max _{j}\left|I_{p}^{j}\right|$. If $\operatorname{width}\left(\mathcal{C}_{p}\right)<\varepsilon s^{-p} / 2$ then

$$
\operatorname{mesh}\left(\mathcal{C}_{p}\right):=\max \left\{\operatorname{diam}(\ell): \ell \in \mathcal{C}_{p}\right\}<\varepsilon,
$$

which shows that $\lim _{\longleftarrow}\left([0,1], T_{S}\right)$ is indeed chainable.

Condition (3) ensures that $\mathcal{C}_{p+1}$ refines $\mathcal{C}_{p}$ (written $\mathcal{C}_{p+1} \preceq \mathcal{C}_{p}$ ).

Definition 2.5 Let $p \in \mathbb{N}_{0}$. A point $x=\left(\ldots, x_{-2}, x_{-1}, x_{0}\right) \in \mathfrak{C}$ is called a $p$-point if $x_{-p-j}=c$ for some $j \in \mathbb{N}_{0}$. For the largest such $j$, the number $L_{p}(x):=j$ is called the $p$-level. In particular, $x_{0}=T_{s}^{p+j}(c)$. The ordered set of all $p$-points of composant $\mathfrak{C}$ is denoted by $E_{p}$, and the ordered set of all $p$-points of $p$-level $l$ by $E_{p, l}$. Given an arc $A \subset \mathfrak{C}$ with successive $p$-points $x^{0}, \ldots, x^{n}$, the $p$-folding pattern of $A$, denoted by $F P_{p}(A)$, is the sequence $F P_{p}(A)=L_{p}\left(x^{0}\right), \ldots, L_{p}\left(x^{n}\right)$. The folding pattern of the composant $\mathfrak{C}$, denoted by $F P(\mathfrak{C})$, is the sequence $L_{p}\left(z^{1}\right), L_{p}\left(z^{2}\right), \ldots$, $L_{p}\left(z^{n}\right), \ldots$, where $E_{p}=\left\{z^{1}, z^{2}, \ldots, z^{n}, \ldots\right\}$ and $p$ is any nonnegative integer. Let $q \in \mathbb{N}, q>p$, and $E_{q}=\left\{y^{1}, y^{2}, \ldots, y^{n}, \ldots\right\}$. Since $\sigma^{q-p}$ is an order-preserving homeomorphism of $\mathfrak{C}$, it is easy to see that, for every $i \in \mathbb{N}, \sigma^{q-p}\left(z^{i}\right)=y^{i}$ and $L_{p}\left(z^{i}\right)=L_{q}\left(y^{i}\right)$. Therefore, the folding pattern of $\mathfrak{C}$ does not depend on $p$.

For the above arc $A$, the projection $\pi_{p}: A \rightarrow[0, s / 2]$ need not be injective, so the folding pattern of $A$ can be very long and $A$ may pass through the same link $\ell^{j}$ of the natural chain $\mathcal{C}_{p}$ many times. If $A^{j}$ is an arc component of $A \cap \ell^{j}$, then we say that $A^{j}$ goes straight through $\ell^{j}$ if $\left.\pi_{p}\right|_{A^{j}}$ is injective; otherwise it turns in $\ell^{j}$. If $A^{j}$ turns in $\ell^{j}$, then $A^{j}$ contains at least one $p$-point.

Definition 2.6 Let $\ell^{0}, \ell^{1}, \ldots, \ell^{k}$ be those links in $\mathcal{C}_{p}$ that are successively visited by an $\operatorname{arc} A=[u, v] \subset \mathfrak{C}$ (hence $\ell^{i} \neq \ell^{i+1}, \ell^{i} \cap \ell^{i+1} \neq \varnothing$ and $\ell^{i}=\ell^{i+2}$ is possible if $A$ turns in $\ell^{i+1}$ ). Let $A^{i} \subset \ell^{i}$ be the corresponding arc components such that $\mathrm{Cl} A^{i}$ are subarcs of $A$. We call the $\operatorname{arc} A$

- $\quad$-link-symmetric if $\ell^{i}=\ell^{k-i}$ for $i=0, \ldots, k$;

- maximal $p$-link-symmetric if it is $p$-link-symmetric and there is no $p$-linksymmetric arc $B \supset A$ and passing through more links than $A$;

- $p$-symmetric if $\pi_{p}(u)=\pi_{p}(v)$ and if for $A \cap E_{p}=\left\{x^{0}, \ldots, x^{n}\right\}$ we have $L_{p}\left(x^{i}\right)=L_{p}\left(x^{n-i}\right)$ for every $i=0, \ldots, n$.

In any of these cases, $k$ is even and the $p$-point of $A^{k / 2}$ with the highest $p$-level is called the center of $A$, and the link $\ell^{k / 2}$ is called the central link of $A$. 
It is easy to see that if $A$ is $p$-symmetric, then $n$ is even and

$$
L_{p}\left(x^{n / 2}\right)=\max \left\{L_{p}\left(x^{i}\right): x^{i} \in A \cap E_{p}\right\} .
$$

Clearly, every $p$-symmetric arc is $p$-link-symmetric as well, but the converse does not hold.

Definition 2.7 Given $p$, let $\left(s_{i}\right)_{i \in \mathbb{N}}$ be the sequence of all $p$-points such that

$$
0 \leq L_{p}(x)<L_{p}\left(s_{i}\right)
$$

for every $p$-point $x \in\left(\alpha, s_{i}\right)$. We call $p$-points satisfying this property salient.

Since for every slope $s>1$ and $p \in \mathbb{N}_{0}$, the sequence $F P(\mathfrak{C})$ starts as $010201 \ldots$, and since by definition $L_{p}\left(s_{1}\right)>0$, we have $L_{p}\left(s_{1}\right)=1$. Also, since $s_{i}=\sigma^{i-1}\left(s_{1}\right)$, $L_{p}\left(s_{i}\right)=i$, for every $i \in \mathbb{N}$. Note that the salient $p$-points depend on $p$ : if $p \geq q$, then the salient $p$-point $s_{i}$ equals the salient $q$-point $s_{i+p-q}$.

Let us extend the notion of folding pattern as follows. A sequence $e_{1}, \ldots, e_{k}$ is the folding pattern of $\left.T^{j}\right|_{H}$ for an interval $H \subset[0,1]$ if $c_{e_{1}}=T^{j}\left(x_{1}\right), \ldots, c_{e_{k}}=T^{j}\left(x_{k}\right)$, where $x_{1}<\cdots<x_{k}$ are the critical points of $T^{j}$ on $H$. (If $0 \in H$, then the folding pattern starts with $*$ by convention, just as $*$ denotes the conventional $p$-level of $\alpha$.) In this extended terminology, the $p$-folding pattern of $\left[\alpha, s_{j+1}\right]$ is the same as the folding pattern of $T^{j}$ on $\left[0, c_{1}\right]$, independently of $p$.

Measured in arc-length, $\bar{d}\left(\alpha, s_{1}\right)=\frac{1}{2} s^{p}$, and since $\sigma\left(s_{i}\right)=s_{i+1}$ we obtain

$$
\bar{d}\left(\alpha, s_{i}\right)=\frac{1}{2} s^{p-1} s^{i} \quad \text { for all } i \geq 1 .
$$

\section{Maximal link-symmetric arcs}

In this section we establish upper bounds for the lengths of $p$-link-symmetric arcs. The Ingram conjecture was previously proved for all tent maps with a (pre)periodic critical point; see Štimac [31]. So we assume from now on that the slope $s$ is such that $c$ is not (pre)periodic. Throughout this section we use the notation $T:=T_{s}, a_{k}:=T^{k}(a)$ for any point or interval (except for the closest precritical points $z_{-k} \in T^{-k}(c)$ in Definition 3.1 below), and $\hat{a}:=1-a$ is the symmetric point around $c$.

Definition 3.1 We call $z_{-k}$ a closest precritical point if $T^{k}\left(z_{-k}\right)=c$ and $T^{k}$ maps $\left[c, z_{-k}\right]$ monotonically onto $\left[c_{k}, c\right]$. Clearly, if $z_{-k}$ is a closest precritical points, so is $\hat{z}_{-k}$. 
Lemma 3.2 There are infinitely many $N$ and closest precritical points $z_{-N}$ such that $\theta_{N}:=\min \left\{\left|c_{i}-c\right|: 0<i \leq N\right\}>\left|z_{-N}-c\right|$.

Proof If $c$ is not recurrent, then $\theta_{n} \not \nrightarrow 0$ and the lemma is trivial. So let us assume that $c$ is recurrent, but obviously not periodic. Let $n$ be such that $\left|c_{n}-c\right|=\theta_{n}$.

If $x \mapsto\left|T^{n}(x)-c\right|$ has a local maximum at $c$, then $T^{n}\left(\left[c, c_{n}\right]\right) \ni c$. Indeed, if this were not the case, then by the choice of $n, T^{n}$ maps $\left[c, c_{n}\right]$ in a monotone fashion into $\left[c, c_{n}\right]$, which is clearly impossible for tent maps with slope $>1$. So in this case, $z_{-n} \in\left[c_{n}, \widehat{c}_{n}\right]$ and the lemma holds with $N=n$.

So assume now that $x \mapsto\left|T^{n}(x)-c\right|$ has a local minimum at $c$. Take $m \in \mathbb{N}$ minimal such that the closest precritical $z_{-m} \in\left[\widehat{c}_{n}, c_{n}\right]$. We will show that $c_{j} \notin\left[\widehat{z}_{-m}, z_{-m}\right]$ for $n<j \leq m$. If $j=m$, then $x \mapsto\left|T^{j}(x)-c\right|$ has a local maximum at $c$, and we can argue as above. So assume by contradiction that $c_{j} \in\left[\widehat{z}_{-m}, z_{-m}\right]$ for some $n<j<m$. If $x \mapsto\left|T^{j}(x)-c\right|$ has a local maximum at $c$, then the closest precritical point $z_{-j}$ satisfies $T^{j}\left(\left[c, z_{-m}\right]\right) \subset T^{j}\left(\left[c, z_{-j}\right]\right)=\left[c_{j}, c\right] \subset\left[\widehat{z}_{-m}, c\right]$ or $\left[c, z_{-m}\right]$. This implies that either $\left[c, z_{-m}\right]$ or $\left[\widehat{z}_{-m}, c\right]$ is mapped monotonically into itself by $T^{j}$, which is impossible. The remaining possibility is that $x \mapsto\left|T^{j}(x)-c\right|$ has a local minimum at $c$. In this case, $T^{j-n}$ maps $\left[z_{-m}, c_{n}\right]$ monotonically onto $\left[w, c_{j}\right]$. If $c \in\left(w, c_{j}\right)$, then $m \in \mathbb{N}$ cannot be minimal such that $T^{m}\left(\left[c, c_{n}\right]\right) \ni c$. If $c \notin\left(w, c_{j}\right)$, then $w \in\left[\widehat{c}_{n}, c_{n}\right] \cap T^{(j-n)-m}(c)$, and since $-m<(j-n)-m<0, m$ is again not minimal such that $T^{m}\left(\left[c, c_{n}\right]\right) \ni c$.

Take $N=m$ and the lemma follows.

Take $N_{0}$ as in Lemma 3.2 and so large that $s^{N_{0}}>100$. Let $N \geq N_{0}$ from Lemma 3.2 be so large that

$$
\delta:=\left|z_{-N}-c\right|<\frac{1}{100}\left|z_{-} N_{0}-c\right| .
$$

Then $\left|c_{n}-c\right| \geq s^{n}\left|z_{-n}-c\right| \geq s^{N_{0}}\left|z_{-N}-c\right|>100 \delta$ for every $N_{0} \leq n \leq N$ by the choice of $N_{0}$ and $\left|c_{n}-c\right|>\left|z_{-N_{0}}-c\right|>100 \delta$ for $n \leq N_{0}$ by the choice of $N$.

Definition 3.3 Given $r \in H$ with $|r-a|,|r-b|>\delta$, we say that $\left.T^{n}\right|_{H}$ is $\varepsilon$-symmetric around $r$, if $\left|T^{n}(r+t)-T^{n}(r-t)\right|<\varepsilon$ whenever both $r-t, r+t \in H$. The point $r$ is called the center of symmetry.

Mostly we will be interested in $\varepsilon$-symmetries around the midpoint of the interval $H$, but we will always specify the center of $\varepsilon$-symmetry, as sometimes there are multiple centers of $\varepsilon$-symmetry. If width $\left(\mathcal{C}_{p}\right)<\varepsilon$ and the arc $J \subset\left[\alpha, s_{k}\right]$ is $p$-link-symmetric, then $\pi_{p+k}: J \rightarrow H:=\pi_{p+k}(J)$ is one-to-one and $\left.T^{k}\right|_{H}$ is $\varepsilon$-symmetric. 
Definition 3.4 We say that $\left.T^{n}\right|_{H}$ is $\varepsilon$-periodic of period $2 \eta$ if

$$
\left|T^{n}(t)-T^{n}(t+2 \eta)\right|<\varepsilon
$$

for all $t, t+2 \eta \in H$.

If $\left.T^{n}\right|_{H}$ is $\varepsilon$-symmetric around two centers $r_{1}$ and $r_{2}$ such that $\left|r_{1}-r_{2}\right|=\eta$, then $\left.T^{n}\right|_{H}$ is $\varepsilon$-periodic with period $2 \eta$. We will explain this fact in more detail in the proof of Proposition 3.6, where it is used several times.

Lemma 3.5 Given $\delta$ as in (3-1), there exists $r_{0}=r_{0}(\delta)$ such that for every interval $\widetilde{J}$ with $\mid \underset{\widetilde{J}}{\tilde{J}} \geq 22 \delta$, there exist $l \leq r_{0} N$ and an interval $J$ with $|J| \geq 18 \delta$ and concentric with $\widetilde{J}$, such that $\left.T^{l}\right|_{J}$ is monotone and $J_{l}:=T^{l}(J) \supset[c-\delta, c+\delta]$.

Proof Let $x$ be the center of $\widetilde{J}$ and take $m \geq 0$ minimal such that $\widetilde{J}_{m} \ni c$; hence $\left.T^{m}\right|_{\tilde{J}}$ is monotone.

Clearly, $m \leq\left(r_{0}-1\right) N$ for some $r_{0} \geq 1$ depending only on $\delta$. If $\partial \widetilde{J}_{m}$ is $\delta$-close to $c$, then we take $J^{\prime} \subset \widetilde{J}$ centered at $x$ and slightly smaller such that $c \in \partial J^{\prime}{ }_{m}$ and $m^{\prime}>m$ minimal such that $J_{m^{\prime}}^{\prime}$ contains $c$ in its interior. Since $\left|J_{m}^{\prime}\right|>20 \delta$, it contains $z_{-N}$ or $\widehat{z}_{-N}$ as in (3-1), and $m^{\prime}-m \leq N$ and $\left|c_{m^{\prime}-m}-c\right| \geq \delta$ by Lemma 3.2.

If at iterate $m^{\prime}$ the other boundary point of $J^{\prime}$ is $\delta$-close to $c$, then $m^{\prime}-m<N$. We take the interval $J^{\prime \prime} \subset J^{\prime}$ centered at $x$ slightly smaller such that $c \in T^{m^{\prime}}\left(\partial J^{\prime \prime}\right)$ and take $m^{\prime \prime}>m^{\prime}$ minimal such that $c$ is an interior point of $T^{m^{\prime \prime}}\left(J^{\prime \prime}\right)$. Since $T^{m^{\prime}}\left(z_{-N}\right) \in T^{m^{\prime}}\left(J^{\prime \prime}\right)$, and by (3-1) again, $m \leq m^{\prime} \leq m^{\prime \prime} \leq m+N$ and $\partial J^{\prime \prime} m^{\prime \prime}$ is not $\delta$-close to $c$. In each case, there is $l \leq r_{0} N$ and $J \in\left\{\widetilde{J}, J^{\prime}, J^{\prime \prime}\right\}$ so that the lemma holds.

For interval $H=:[a, b]$ with center $x$ we formulate the following property:

$$
c \in H \quad \text { and } \quad \delta<\min \{|c-a|,|c-b|,|c-x|\} .
$$

Proposition 3.6 Assume that $s \in[1,2]$ is such that $c$ is not (pre)periodic. There exists $\varepsilon>0$ such that if $H$ satisfies (3-2), then $\left.T^{n}\right|_{H}$ is not $\varepsilon$-symmetric around its midpoint $x$ for any $n \in \mathbb{N}_{0}$.

Proof We will prove Proposition 3.6 using the induction hypothesis:

$\left(\mathrm{IH}_{n}\right) \quad$ If $H$ satisfies (3-2), then $\left.T^{n}\right|_{H}$ is not $\varepsilon$-symmetric around $x$.

Take $N_{0}, N$ and $\delta$ as in (3-1), $r_{0}$ as in Lemma 3.5 and $H$ that satisfies (3-2). 
Let $\varepsilon \in(0, \delta)$ be so small that

$$
\varepsilon<\min \left\{\left|c_{i}-c_{j}\right|: 0 \leq i<j \leq\left(2+r_{0}\right) N\right\} .
$$

Since $c$ lies off-center in $H$ by at least $\delta$, by the choice of $\varepsilon,\left(\mathrm{IH}_{k}\right)$ holds for all $k \leq\left(2+r_{0}\right) N$. Assume now that $\left(\mathrm{IH}_{j}\right)$ holds for all $j<n$. We will prove $\left(\mathrm{IH}_{n}\right)$, but first, continuing with the interval $\widetilde{J}$ of Lemma 3.5, we prove the following lemma.

Lemma 3.7 Let $\widetilde{J}$ be an interval of length $|\widetilde{J}| \geq 22 \delta$ centered at $c_{k}$ for some $1 \leq$ $k \leq 2 N$. If $T^{j} \mid \widetilde{J}$ is $\varepsilon$-symmetric around $c_{k}$ for some $0 \leq j \leq n$, then the interval $J_{l}:=T^{l}(J)$ from Lemma 3.5 satisfies condition (3-2).

Proof We know already from Lemma 3.5 that $J_{l} \supset[c-\delta, c+\delta]$. Hence if (3-2) fails, then $\eta:=\left|c_{k+l}-c\right| \leq \delta$. Since $\left.T^{l}\right|_{J}$ is monotone, $j>l$. Therefore $\left.T^{j-l}\right|_{J_{l}}$ is $\varepsilon$-symmetric around $c_{k+l}$ and symmetric around $c$, and it follows that $\left.T^{j-l}\right|_{J_{l}}$ is $\varepsilon$-periodic with period $2 \eta$. Indeed, by symmetry around $c,\left.T^{j-l}\right|_{J_{l}}$ is $\varepsilon$-symmetric around the symmetric point $\widehat{c}_{k+l}$ (see Figure 1). Hence $\left.T^{j-l}\right|_{J_{l}}$ must also be $\varepsilon-$ symmetric around the points $c \pm 2 \eta$, which are the reflections of $c$ in $c_{k+l}$ and $\widehat{c}_{k+l}$, etc. Extending these symmetries, we see that $\left|T^{j-l}(t)-T^{j-l}(t+2 \eta)\right|<\varepsilon$ for all $t, t+2 \eta$ in $J_{l}$, so $\left.T^{j-l}\right|_{J_{l}}$ is $\varepsilon$-periodic with period $2 \eta$. Even more, $\left.T^{j-l}\right|_{J_{l}}$ is $\varepsilon$-symmetric around $c+2 i \eta$ on every separate subarc $P_{i}:=[c+(2 i-1) \eta, c+(2 i+1) \eta] \subset J_{l}$.

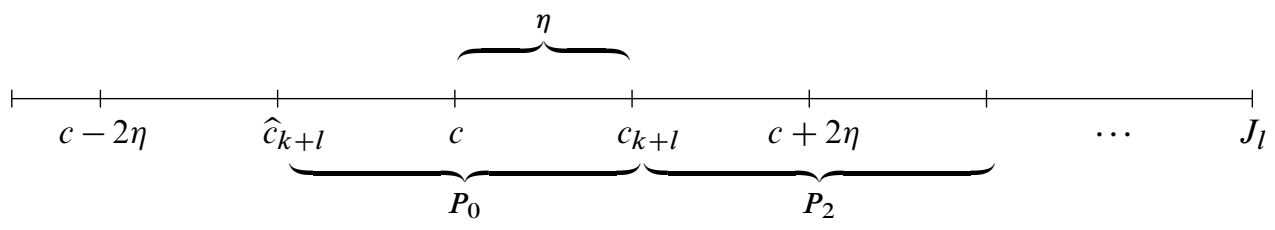

Figure 1: $\left.T^{j-l}\right|_{J_{l}}$ is $\varepsilon$-symmetric around $c_{k+l}$ and symmetric around $c$, implying that $\left.T^{j-l}\right|_{J_{l}}$ is $\varepsilon$-periodic with period $2 \eta$.

Recall that $1 \leq k \leq 2 N$ and $l \leq r_{0} N$, so we have $\eta>\varepsilon$ by the choice of $\varepsilon$ in (3-3). Since $\left|J_{l}\right| \geq 18 \delta=18\left|z_{-N}-c\right|$, one of the components of $J_{l} \backslash\{c\}$, say the one containing $z_{-N}>c$, has length $\geq 9 \delta$. We can take $r \leq N$ minimal such that $z_{-r} \in[c+\delta, c+8.9 \delta]$. Take $i \in \mathbb{Z}$ such that if

$$
z_{-r} \in \begin{cases}(c, c+4.3 \delta] & \text { then } c+2 i \eta \in\left(z_{-r}+0.1 \delta, z_{-r}+2.1 \delta\right), \\ (c+4.3 \delta, c+8.9 \delta] & \text { then } c+2 i \eta \in\left(z_{-r}-2.1 \delta, z_{-r}-0.1 \delta\right) .\end{cases}
$$

Let $H \subset J_{l}$ be the longest interval centered at $x:=c+2 i \eta$ on which $\left.T^{r}\right|_{H}$ is monotone. Then $H \ni z_{-r}$, and $\left.T^{j-l}\right|_{H}$ and $\left.T^{j-l-r}\right|_{H_{r}}$ are $\varepsilon$-symmetric around $x$. We will show that $H_{r}$ satisfies (3-2). Indeed, since $\left|z_{-r}-c\right| \leq 9 \delta<\left|z_{N_{0}}-c\right| / 10$ (so 
$\left.r>N_{0}\right)$ by (3-1) and $\left|x-z_{-r}\right| \geq \delta / 10$, we have $\left|x_{r}-c\right|=s^{r}\left|x-z_{-r}\right| \geq 2^{N_{0} / 2} \delta / 10>\delta$. If $\left|z_{-r}-\partial H\right| \geq \delta / 10$, then $\left|c-\partial H_{r}\right|>\delta$ for the same reason. If on the other hand there is a point $y \in \partial H$ such that $\left|y-z_{-r}\right|<\delta / 10$, then $y$ has to be a precritical point. By the choice of $r, y=z_{-r^{\prime}} \in(c+8.9 \delta, c+9 \delta]$ for some $r^{\prime}<r$. By the choice of $N$ and Lemma 3.2, $\left|y_{r}-c\right|=\left|c_{r-r^{\prime}}-c\right| \geq \delta$.

This shows that $H_{r}$ satisfies (3-2), but also $\left.T^{j-l-r}\right|_{H_{r}}$ is $\varepsilon$-symmetric around $x_{r}$, and this contradicts $\left(\mathrm{IH}_{j-l-r}\right)$, proving this lemma.

Combining the induction hypothesis $\left(\mathrm{IH}_{n}\right)$ and Lemma 3.7, we have proved the following stronger property.

Corollary 3.8 If $\tilde{J}$ is centered at $c_{k}$ for some $1 \leq k \leq 2 N$ and $|\widetilde{J}| \geq 22 \delta$, then $T^{j} \mid \tilde{J}$ is not $\varepsilon$-symmetric around $c_{k}$ for $j \leq n$.

Now we continue the induction on $n$ and assume by contradiction that $\left.T^{n}\right|_{H}$ is $\varepsilon-$ symmetric around midpoint $x$ for some $H$ satisfying (3-2) and for $\varepsilon$ satisfying (3-3). Let $\left[a^{\prime}, b^{\prime}\right]:=H^{\prime} \subset H$ be centered around $x$ such that $c \in \partial H^{\prime}$. Assume without loss of generality that $c=a^{\prime}$ is the left endpoint of $H^{\prime}$, and let $L$ and $R$ be intervals of length $\delta$ at the left and right side adjacent to $H^{\prime}$. Since $\left|H^{\prime}\right| \geq \delta$, so $H^{\prime} \ni z_{-N}$ or $\widehat{z}_{-N}$, there is $0<k \leq N$ minimal such that $c \in H^{\prime}{ }_{k}$. Clearly $\left|H^{\prime}{ }_{k}\right|>\left|L_{k}\right|=\left|R_{k}\right| \geq 100 \delta$. We distinguish four cases:

Case I $H^{\prime}{ }_{k}$ satisfies (3-2). Then by $\left(\mathrm{IH}_{n-k}\right),\left.T^{n-k}\right|_{H^{\prime}{ }_{k}}$ cannot be $\varepsilon$-symmetric around $x$, and neither can $\left.T^{n}\right|_{H^{\prime}}$ or $\left.T^{n}\right|_{H}$.

Case II $\left|x_{k}-c\right|<\delta$; see Figure 2 (left). If the length of the interval $T^{n-k}\left(\left[x_{k}, c\right]\right)$ exceeds $\varepsilon$, then since $T^{n-k}$ is also symmetric around $c, T^{n-k}$ must be $\varepsilon$-symmetric on $H^{\prime}{ }_{k}$ both with center $x_{k}$ and with center $\hat{x}_{k}$, and therefore $\varepsilon$-periodic on $H^{\prime}$ with period $2 \eta:=2\left|x_{k}-c\right|$. We use the same argument as in the proof of Lemma 3.7: $T^{n-k}$ is $\varepsilon$-symmetric on each interval $P_{i}:=[c+(2 i-1) \eta, c+(2 i+1) \eta]$ for each $i \in \mathbb{Z}$ such that $P_{i} \subset H^{\prime}{ }_{k}$. Since $\left|H^{\prime}{ }_{k}\right| \geq 100 \delta \geq 100 \eta, P_{i} \subset H^{\prime}{ }_{k}$ for at least $-25 \leq i \leq 25$. Take $r \leq N$ minimal such that $\left[z_{-r}-\delta / 10, z_{-r}+\delta / 10\right] \subset H^{\prime}{ }_{k}$, and $i \in \mathbb{Z}$ as in (3-4), and $H^{\prime \prime} \subset H^{\prime}$ such that $H^{\prime \prime}{ }_{k}$ is the maximal interval centered at $c+2 i \eta$ on which $T^{r}$ is monotone. As before, $\left.T^{n-(k+r)}\right|_{H^{\prime \prime}{ }_{k+r}}$ is the $\varepsilon$-symmetric but $H^{\prime \prime}{ }_{k+r}$ satisfies (3-2). This would contradict $\left(\mathrm{IH}_{n-(k+r)}\right)$, so it cannot occur.

If on the other hand the length of $T^{n-k}\left(\left[x_{k}, c\right]\right)$ is less than $\varepsilon$, then we might as well have chosen $x$ such that $x_{k}=c$. This means that the intervals $L_{k+1}$ and $R_{k+1}$ are adjacent; see Figure 2 (bottom left). More precisely, they are adjacent except for an error which does not show at $\varepsilon$-scale under the iterate $T^{n-(k+1)}$, so by a negligible 

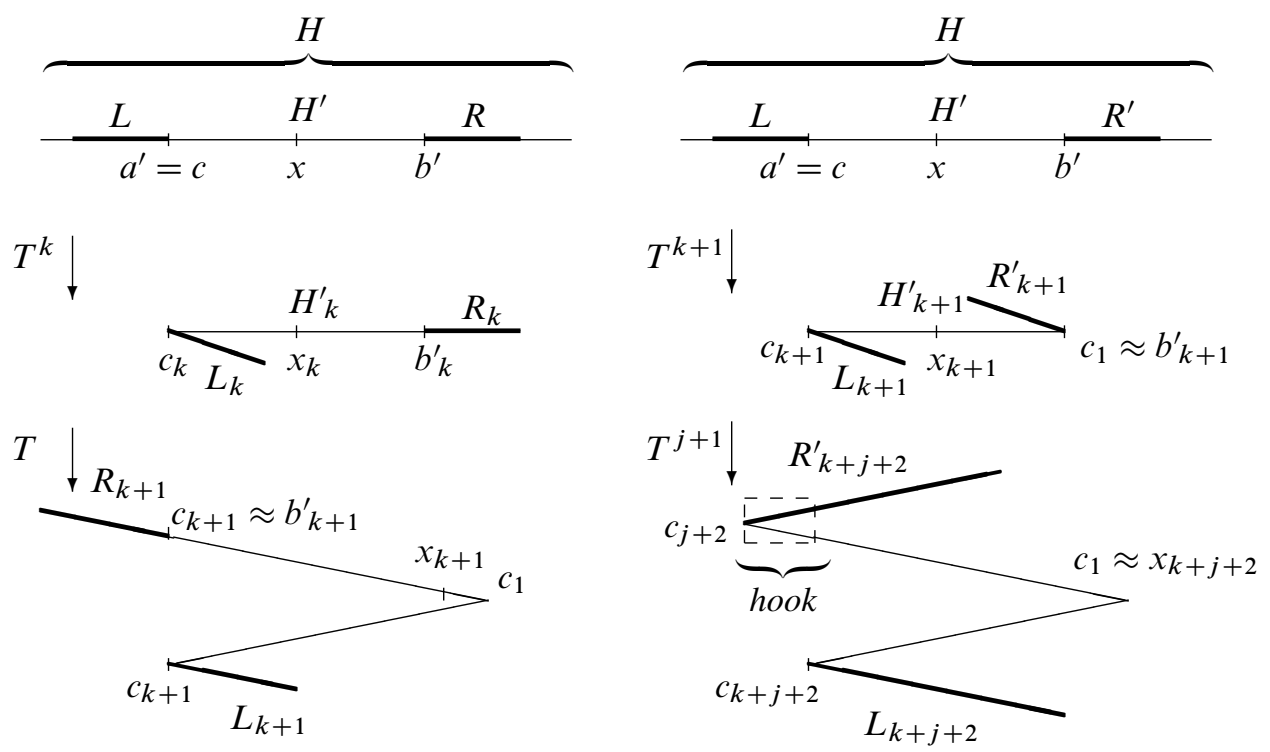

Figure 2: An illustration of Cases II (left) and IV (right)

adjustment, we can assume that they form an interval of length $\geq 100 \delta$ with center $c_{k+1}$. Since $k+1 \leq 2 N$, Corollary 3.8 implies that $\left.T^{n-(k+1)}\right|_{L_{k+1} \cup R_{k+1}}$ and hence $\left.T^{n}\right|_{H}$ are not $\varepsilon$-symmetric around $x$.

Case III $\left|a_{k}^{\prime}-c\right|<\delta$. Since $k \leq N$, the choice of $\delta$ renders this impossible.

Case IV $\left|b^{\prime}{ }_{k}-c\right|<\delta$; see Figure 2 (right). Replace $R$ by the largest interval $R^{\prime} \subset$ $\overline{H \cup R}$ with $R^{\prime} \cap R \neq \varnothing$ such that $c \in \partial R_{k}^{\prime}$ and $\left.T^{k}\right|_{R^{\prime}}$ is monotone. If $c \in \partial R_{l}^{\prime}$ for some $0 \leq l<k$, then $R_{k}^{\prime}=\left[c, c_{k-l}\right]$, so $\left|R_{k}^{\prime}\right| \geq \delta$ by Lemma 3.2. Also rename $H^{\prime} \backslash R^{\prime}$ to $H^{\prime}$. Hence $\left.T^{k+1}\right|_{L \cup H^{\prime} \cup R^{\prime}}$ has three branches, $s \delta \leq\left|R_{k+1}^{\prime}\right|$ and $100 \delta \leq$ $\left|L_{k+1}\right| \leq\left|H_{k+1}^{\prime}\right|$.

Let $j>0$ be minimal such that $T^{k+j+1}\left(H^{\prime}\right) \ni c$. If $H_{k+j+1}^{\prime}=\left[c_{k+j+1}, c_{j+1}\right]$, which is centered at $x_{k+j+1}$, satisfies (3-2), then we can invoke $\left(\mathrm{IH}_{n-(k+j+1)}\right)$, so assume that this is not the case. Since $|L| \geq \delta$, so $L \ni z_{-N}$ or $\widehat{z}_{-N}$, we have $j \leq k+j+1 \leq N$. Therefore both $\left|c_{j+1}-c\right|>\delta$ and $\left|c_{k+j+1}-c\right| \geq \delta$.

Thus if (3-2) fails, we must have $\left|x_{k+j+1}-c\right|<\delta$. If in the remaining $n-(k+j+1)$ iterates, the arc $\left[x_{k+j+1}, c\right]$ grows to length $>\varepsilon$, then, as in Case II, $\left.T^{n}\right|_{H^{\prime}}$ must contain a large $\varepsilon$-periodic arc, to which we apply the same argument as in Case II (ie the argument of Lemma 3.7). The remaining possibility is that $x_{k+j+1}$ is so close to $c$ that on an $\varepsilon$-scale, we may as well assume that $x_{n+k+1}=c$. 
Both $c_{k+j+2}=a_{k+j+2}^{\prime}$ and $c_{j+2} \approx b_{k+j+2}^{\prime}$ are local minima of $\left.T^{k+j+2}\right|_{L \cup H^{\prime} \cup R^{\prime}}$; see Figure 2 (bottom right). Assume without loss of generality that $c_{j+2}<c_{k+j+2}$, so $R_{k+j+2}^{\prime}$ has a small extra hook before joining up with $L_{k+j+2}$. As we assumed that $\left.T^{n}\right|_{H}$ is $\varepsilon$-symmetric around $x$, the effect of this hook needs to be " $\varepsilon$-repeated" near $a^{\prime}$ in $L$. But $L_{k+j+2}$ and $R_{k+j+2}^{\prime}$ overlap, so in $R^{\prime}$, the same effect needs to be $\varepsilon$-repeated next to the first hook. Continuing this way, we find that $T^{n-(k+j+2)}$ is $\varepsilon$-periodic over the entire length of $R_{k+j+2}^{\prime}$.

Take $i$ minimal such that $R^{\prime \prime}:=T^{i}\left(R_{k+j+2}^{\prime}\right) \ni c$. Since $\left|R_{k}^{\prime}\right| \geq \delta$ we have

$$
j+i+2<N, \quad\left|R^{\prime \prime}\right| \geq 100 \delta \quad \text { and } \quad\left|\partial R^{\prime \prime}-c\right| \geq \delta .
$$

Therefore $\left.T^{n-(k+j+i+2)}\right|_{R^{\prime \prime}}$ is $\varepsilon$-periodic of period $2 \eta$, where the length of the hook after $i$ more iterates is $\eta:=\left|c_{j+i+2}-c_{k+j+i+2}\right|>\varepsilon$, because $k+j+i+2 \leq 2 N$ and by the choice of $\varepsilon$ in (3-3). If $\eta<10 \delta<\left|R^{\prime \prime}\right| / 10$, then $\left.T^{n-(k+j+i+2)}\right|_{R^{\prime \prime}}$ is $\varepsilon$-periodic with at least 5 adjacent intervals $P$ of length $2 \eta$ around the center of which $\left.T^{n-(k+j+i+2)}\right|_{R^{\prime \prime}}$ is $\varepsilon$-symmetric. So we can find a new interval $H^{\prime \prime} \subset R^{\prime \prime}$ centered around the center of one of these $P$ s such that $H^{\prime \prime}$ satisfies (3-2). But this contradicts $\left(\mathrm{IH}_{n-(k+j+i+2)}\right)$.

If $\eta \geq 10 \delta$, then we let $H^{\prime \prime}$ be the arc of length $22 \delta$ centered at $c_{k+j+i+2}$. Again, since $k+j+i+2 \leq 2 N$, the iterate $T^{n-(k+j+i+2)}$ cannot be $\varepsilon$-symmetric on $H^{\prime \prime}$ around $c_{k+j+i+2}$ by Corollary 3.8. But then the assumed $\varepsilon$-symmetry of $\left.T^{n}\right|_{H}$ does not extend beyond $H^{\prime}$, and Case IV follows.

This proves the inductive step and the proof of Proposition 3.6 is complete.

Let $\kappa:=\min \left\{i \geq 3: c_{i} \geq c\right\}$. Then $\kappa<\infty$ provided $1<s<2$. Let

$$
\cdots<c_{-3}<c_{-2}<c_{-1}<c_{0}=c
$$

be the successive precritical points on the left of $c$ with $T^{j}\left(c_{-j}\right)=c$. Since we have $c_{\kappa-1}<c<c_{\kappa}$, then $c_{2-\kappa}<c_{2}<c_{3-\kappa}$. Let $\delta=\left|z_{-N}-c\right|$ as in (3-1) be so small (ie $N$ as in Lemma 3.2 so large) that

$$
\delta<\frac{1}{30} \min \left\{\left|c_{-1}-c_{-2}\right|,\left|c_{-1}-\widehat{c}_{1}\right|,\left|c_{2}-c_{2-\kappa}\right|\right\}
$$

where $\widehat{c}_{1}=1-c_{1}=1-s / 2$. Assume that $s \in[1,2]$ is such that $c$ is not (pre)periodic, and take $\varepsilon$ is as in (3-3) in the proof of Proposition 3.6.

Let $\left(A_{i}\right)_{i \in \mathbb{N}}$ be the sequence of maximal $p$-link-symmetric arcs with center $s_{i}$ for every $i \in \mathbb{N}$. Recall that $\left(s_{i}\right)_{i \in \mathbb{N}}$ is the sequence of salient $p$-points (see Definition 2.7) and that width $\left(\mathcal{C}_{p}\right):=\max _{j}\left|I_{p}^{j}\right|$. 
Lemma 3.9 If width $\left(\mathcal{C}_{p}\right)<\varepsilon$, then $A_{i}$ contains exactly $\kappa$ salient $p$-points for each $i \geq \kappa-1$, namely $s_{i-\kappa+2}, s_{i-\kappa+3}, \ldots, s_{i+1}$, and $s_{i-\kappa+2}$ is an interior point of $A_{i}$.

Proof Let $H$ be the interval centered at $c_{2}$ such that $c$ is the left endpoint of $H_{\kappa-2}:=T^{\kappa-2}(H)$. Then $|H| \geq 22 \delta$ by the choice of $\delta$, so by Proposition 3.6 and Lemma 3.7 in particular, $\left.T^{p+i-1}\right|_{H}$ cannot be $\varepsilon$-symmetric around $c_{2}$.

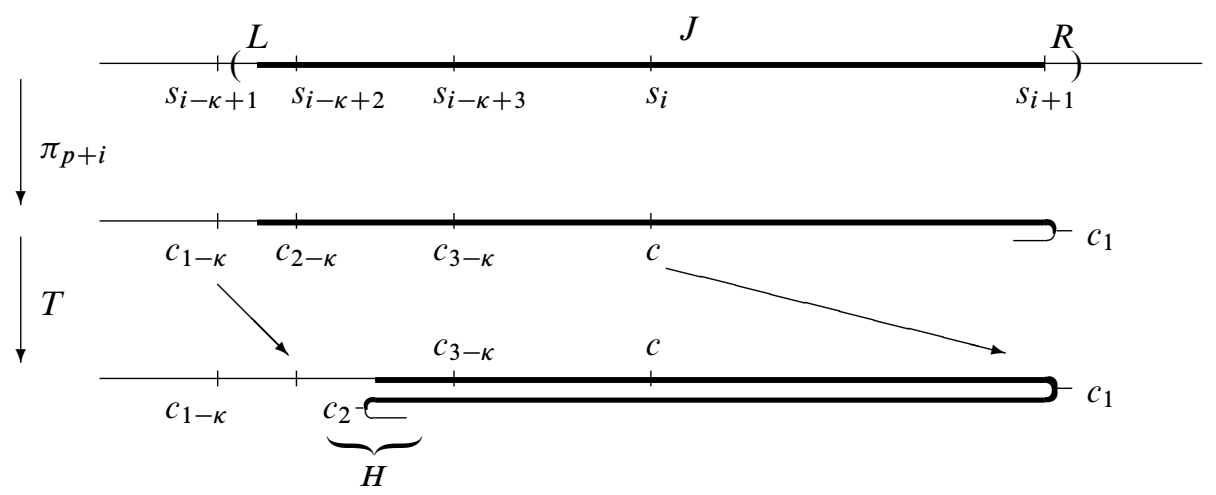

Figure 3: The arc $J$ and its image under $\pi_{p+i}$ and $T \circ \pi_{p+i}=\pi_{p+i-1}$

Let $J=\left[x, s_{i+1}\right]$ be such that $J \ni s_{i}$ and $\bar{d}\left(s_{i}, s_{i+1}\right)=\bar{d}\left(x, s_{i}\right)$, where $\bar{d}$ is defined in Definition 2.3. Then $\pi_{p+i-1}$ maps $J$ in a 2-to-1 fashion onto $\left[c_{2}, c_{1}\right]$, with $\pi_{p+i-1}\left(s_{i}\right)=c_{1}$ and $\pi_{p+i-1}\left(s_{i+1}\right)=c_{2}$. Therefore $J$ is $p$-symmetric and also $p$-link-symmetric around $s_{i}$. Since $c_{2-\kappa}<c_{2}<c_{3-\kappa}$, we have $\pi_{p+i-1}(J) \not \ngtr c_{2-\kappa}$. Extend $J$ on either side by equally long arcs $L$ and $R$ such that $\pi_{p+i-1}(L \cup R)=H$; see Figure 3. Since $\left.T^{i-1}\right|_{H}$ is not $\varepsilon$-symmetric around $c_{2}, A_{i} \not \supset L \cup J \cup R$ provided width $\left(\mathcal{C}_{p}\right)<\varepsilon$. Hence $A_{i} \not \ngtr s_{i-\kappa+1}$ as claimed.

Remark 3.10 The bound $\kappa$ in this lemma is not sharp if $T_{s}$ has a periodic critical point. For example, for the tent map with $c_{2}<c=c_{3}<c_{1}$, the folding pattern is

$$
\begin{aligned}
& \text { maximal } p \text {-symmetric }
\end{aligned}
$$

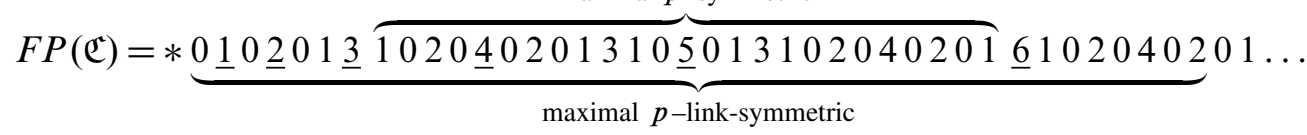

where $p$-levels of salient $p$-points are underlined and $*$ denotes the conventional $p$-level of $\alpha$. Since $c$ has period 3, so $c_{a}=c_{a+3 b}$ for all $a, b \in \mathbb{N}, p$-link-symmetric arcs can be longer than $p$-symmetric arcs. Indeed, the maximal $p$-symmetric arc centered at salient point $s_{5}$ stretches from $s_{3}$ to $s_{6}$, while maximal $p$-link-symmetric arc centered at $s_{5}$ stretches almost from $\alpha$ to some point with $p$-level 2 . This property 
holds for all salient points: the maximal $p$-link-symmetric arc around $s_{i}$ contains $s_{j}$ for all $j \leq i+1$.

A preperiodic example is $s=2$, ie $\lim \left([0,1], T_{s}\right)$ is the Knaster continuum.

Lemma 3.11 Assume that width $\left(\mathcal{C}_{p}\right)<\varepsilon$ and fix $i \in \mathbb{N}, i>\kappa-1$, and let $\ell^{i}$ and $\ell^{i-1}$ be the links of $\mathcal{C}_{p}$ containing $s_{i}$ and $s_{i-1}$ respectively. Let $y$ be such that $s_{i-1} \prec y \prec s_{i}$ and $y$ is not contained in the same arc-component of $\ell^{i}$ as $s_{i}$, nor in the same arccomponent of $\ell^{i-1}$ as $s_{i-1}$. Then the maximal $p$-link-symmetric arc $J$ with center $y$ contains at most one salient $p$-point, and $J \subset A_{i}$.

Proof Let $\cdots<c_{-2}<c_{-1}<c_{0}=c$ be the successive precritical points to the left of $c$ with $T^{j}\left(c_{-j}\right)=c$. Since $A_{i}$ contains $s_{i+1}$ and its symmetric point around $s_{i}$ (at least as boundary points), we have $\pi_{p+i}\left(A_{i}\right) \supset\left[\hat{c}_{1}, c_{1}\right] \supset\left[c_{-1}, c\right]$. Let $H:=\pi_{p+i}(J)$ with center $x:=\pi_{p+i}(y) \in\left[c_{-1}, c\right]$. Assume by contradiction that $J$ contains two salient $p$-points, or that $J \not \subset A_{i}$. Then $|H| \geq 22 \delta$ by the choice of $\delta$ in (3-5).

Let $w:=\left(c_{-1}+c\right) / 2$. We distinguish four cases.

(1) $c-\delta<x<c$. If $\left|T^{i}([x, c])\right| \leq \varepsilon$, then we cannot " $\varepsilon$-distinguish" $x$ from $c$, violating our assumption that $y$ and $s_{i}$ do not belong to the same arc-component of the same link. If $\left|T^{i}([x, c])\right|>\varepsilon$, then $T^{i}$ is $\varepsilon$-symmetric on $H$ with centers $x$ and $c$, so $T^{i}$ is $\varepsilon$-periodic on $H$ with period $2|x-c|$. This leads to a contradiction by the argument of the proof of Lemma 3.7.

(2) $w \leq x \leq c-\delta$. Then $H$ satisfies (3-2), so by Proposition 3.6, $\left.T^{i}\right|_{H}$ cannot be $\varepsilon$-symmetric around $x$.

(3) $c_{-1}+\delta / s \leq x<w$. Then by assumption $H$ contains one of $c, c_{-2}$ or $\widehat{c}_{1}$ (whence $|H| \geq 22 \delta)$, and hence $T\left(H \cap\left[c_{-2}, c\right] \cap\left[\widehat{c}_{1}, c\right]\right)$ satisfies (3-2), so $\left.T^{i}\right|_{H}$ cannot be $\varepsilon$-symmetric around $x$ by Proposition 3.6.

(4) $c_{-1}<x<c_{-1}+\delta / s$. If $\left|T^{i}\left(\left[c_{-1}, x\right]\right)\right| \leq \varepsilon$, then we cannot " $\varepsilon$-distinguish" $x$ from $c$, violating the assumption that $y$ and $s_{i-1}$ are not contained in the same arc component of $\ell^{i-1}$. If $\left|T^{i}\left(\left[c_{-1}, x\right]\right)\right|>\varepsilon$ and again, $H$ by assumption contains one of $c, c_{-2}$ or $\hat{c}_{1}$ (so $|H| \geq 22 \delta$ ), then $T^{i-1}$ is $\varepsilon$-periodic on $T(H)$ which again leads to a contradiction by the argument of the proof of Lemma 3.7.

This proves the lemma. 


\section{Link-symmetric arcs and homeomorphisms}

In this section we study the action of homeomorphisms

$$
h: \underset{\lim }{\longleftarrow}\left([0,1], T_{s^{\prime}}\right) \longrightarrow \lim _{\longleftarrow}\left([0,1], T_{s}\right)
$$

on salient $q$-points and $q$-points in general. Let $q, p, g \in \mathbb{N}_{0}$ be such that

$$
h\left(\mathcal{C}_{q}\right) \preceq \mathcal{C}_{p} \preceq h\left(\mathcal{C}_{g}\right) .
$$

Recall that we assumed the slopes $s^{\prime}$ and $s$ to be such that the critical points $c^{\prime}$ and $c$ are not (pre)periodic. Clearly $h$ maps the zero-composant $\mathfrak{C}^{\prime}$ of $\lim \left([0,1], T_{s^{\prime}}\right)$ to the zero-composant $\mathfrak{C}$ of $\lim \left([0,1], T_{S}\right)$, and in particular the endpoint $\alpha^{\prime}$ of $\mathfrak{C}^{\prime \prime}$ to the

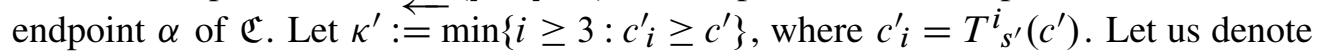
the salient $q$-points (ie associated with $\mathcal{C}_{q}$ ) by $s_{i}^{\prime}$ and the salient $g$-points by $s^{\prime \prime}{ }_{i}$. Therefore, a salient $q$-point $s_{i}^{\prime}$ is the same as a salient $g$-point $s^{\prime \prime}{ }_{i+q-g}$. Similarly, let $A_{i}^{\prime}$ be the maximal $q$-link-symmetric arc centered at $s_{i}^{\prime}$ while as before, $A_{i}$ denotes the maximal $p$-link-symmetric arc centered at $s_{i}$

Since $A_{i}^{\prime}$ is $q$-link-symmetric, and $h\left(\mathcal{C}_{q}\right) \preceq \mathcal{C}_{p}$, the image $D_{i}:=h\left(A_{i}^{\prime}\right) \subset \mathfrak{C}$ is $p$-link-symmetric and therefore has a well-defined center, we denote it as $m_{i}$, and a well-defined central link $\ell_{p}$ (see Definition 2.6). In fact, $h\left(s_{i}^{\prime}\right)$ and $m_{i}$ belong to the central link $\ell_{p}$ and $m_{i}$ is the $p$-point with the highest $p$-level of all $p$-points of the arc component of $\ell_{p}$ which contains $h\left(s_{i}\right)$. Let $M_{i}:=L_{p}\left(m_{i}\right)$.

Theorem 4.1 $M_{i+1}=M_{i}+1$ for all sufficiently large integers $i \in \mathbb{N}$.

Proof Without loss of generality we can assume that $s^{\prime} \geq s$, so that $\kappa^{\prime} \geq \kappa$. We prove first that if $N \geq \kappa$ is so large that $m_{N}$ lies beyond the $\kappa$-th salient $p$-point of $\mathfrak{C}$, then $L_{p}(y)<M_{N}$, for every $y \in\left(\alpha, m_{N}\right)$; ie $m_{N}$ is salient.

Assume by contradiction that there exists $y \in\left(\alpha, m_{N}\right)$ such that $L_{p}(y) \geq M_{N}$. By taking $L_{p}(y)$ maximal with this property, we can assume that $y=s_{j-1} \prec m_{N} \prec s_{j}$ for some $j>\kappa$. More precisely, $m_{N}$ is not contained in the same arc-component of the link containing $s_{j-1}$ as $s_{j-1}$, and similarly for $s_{j}$. Lemma 3.11 implies that $D_{N}$ contains at most one salient $p$-point and that $D_{N} \subset A_{j}$. Let us denote by $B$ the $p-$ link-symmetric arc such that $s_{j}$ is the center of $B, D_{N} \subset B \subset A_{j}$ and $\partial D_{N} \cap \partial B \neq \varnothing$ (see Figure 4). Since $\mathcal{C}_{p} \preceq h\left(\mathcal{C}_{g}\right)$, the arc $B^{\prime \prime}=\sigma^{q-g} \circ h^{-1}(B)$ is $g$-link-symmetric and contains the arc $\sigma^{q-g} \circ h^{-1}\left(D_{N}\right)=\sigma^{q-g}\left(A_{N}^{\prime}\right)$. The center $z^{\prime \prime}$ of $B^{\prime \prime}$ is the center of the arc component of the central link $\ell_{g}$ of $B^{\prime \prime}$ containing $\sigma^{q-g} \circ h^{-1}\left(s_{j}\right)$. By Lemma 3.9, $A^{\prime}{ }_{N}$ contains $\kappa^{\prime}$ salient $q$-points $s^{\prime}{ }_{N-\kappa^{\prime}+2}, \ldots, s_{N}^{\prime}, s_{N+1}^{\prime}$. 


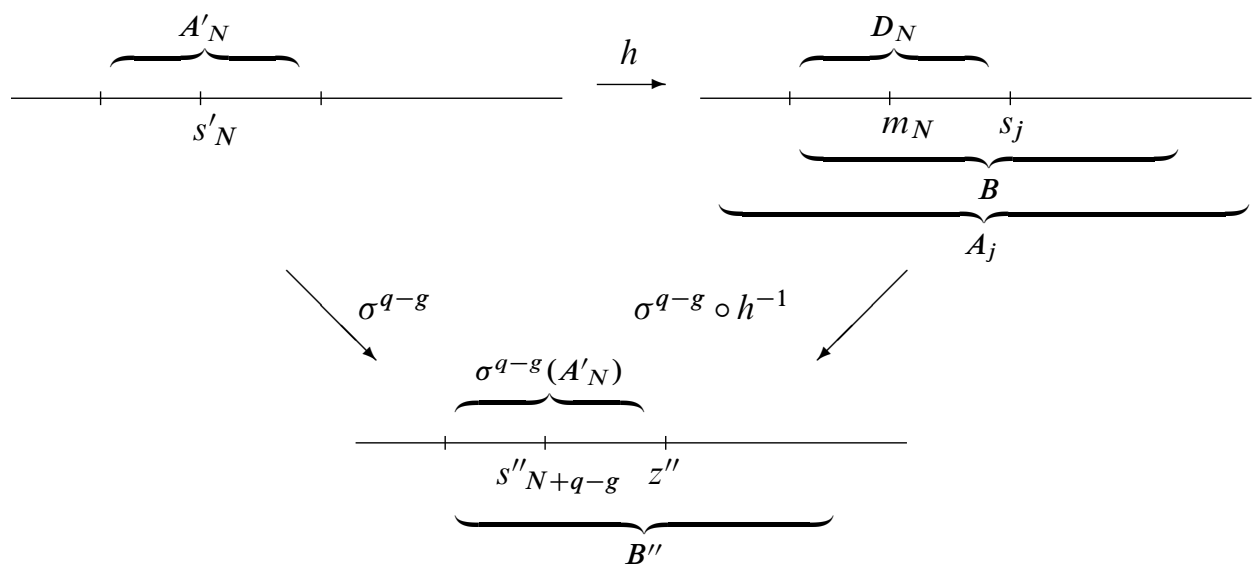

Figure 4: The relations between relative salient points and $\operatorname{arcs}$ in $\mathcal{C}_{q}$ (left), $\mathcal{C}_{p}$ (right), and $\mathcal{C}_{g}$ (bottom)

The map $\sigma^{q-g}$ maps the $\kappa^{\prime}$ salient $q$-points $s_{i}^{\prime} \in A_{N}^{\prime}$ to the $\kappa^{\prime}$ salient $g$-points $s^{\prime \prime}{ }_{i+q-g} \in \sigma^{q-g}\left(A_{N}^{\prime}\right)$, and $B^{\prime \prime}$ contains at least these $\kappa^{\prime}$ salient $g$-points. If the center $z^{\prime \prime}$ of $B^{\prime \prime}$ is not salient, then $B^{\prime \prime}$ contains at most one salient $g$-point by Lemma 3.11, so we have a contradiction. Otherwise, if $z^{\prime \prime}$ is salient, then even if $z^{\prime \prime}$ is the right-most salient $g$-point of $\sigma^{q-g}\left(A_{N}^{\prime}\right)$, then still $B^{\prime \prime}$ contains $\kappa^{\prime}-1$ salient $g$-points on the left of the center $z^{\prime \prime}$, contradicting Lemma 3.9. Therefore, $m_{N}$ is salient.

Let us consider the arc $D_{N+\kappa^{\prime}-2}=h\left(A_{N+\kappa^{\prime}-2}^{\prime}\right)$. Since $L_{q}\left(s_{i+1}^{\prime}\right)-L_{q}\left(s_{i}^{\prime}\right)=1$, the arc $\left[s_{i}^{\prime}, s_{i+1}^{\prime}\right]$ contains a $q$-point of every $q$-level less than $i$, so contains $q$-points of $q$-levels 1 and 2 . Therefore, $\pi_{q}\left(\left[s_{i}^{\prime}, s_{i+1}^{\prime}\right]\right)=\left[c_{2}, c_{1}\right]$. Note that two different points from $s_{N}^{\prime}, \ldots, s_{N+\kappa^{\prime}-1}^{\prime} \in A_{N+\kappa^{\prime}-2}^{\prime}$ can be mapped into the same link, say $\ell_{p}$ of $\mathcal{C}_{p}$, but cannot be mapped into the same arc component of $\ell_{p}$. Indeed, if $h\left(\left[s_{i}^{\prime}, s_{i+1}^{\prime}\right]\right) \subset A$, where $A$ is a arc component of $\ell_{p}$, then $h\left(\mathcal{C}_{q}\right) \subset \ell_{p}$, a contradiction. Therefore, $s_{M_{N}}, \ldots, s_{M_{N+\kappa^{\prime}-1}}$ are all different.

So, the arc $D_{N+\kappa^{\prime}-2}$ is $p$-link-symmetric and contains at least $\kappa^{\prime}$ salient $p$-points, $s_{M_{N}}, \ldots, s_{M_{N+\kappa^{\prime}-1}}$. By Lemma 3.9, the maximal $p$-link-symmetric arc $A_{M_{N+\kappa^{\prime}-2}}$ centered at the salient $p$-point $s_{M_{N+\kappa^{\prime}-2}}$ contains $\kappa$ salient $p$-points, namely

$$
s_{M_{N+\kappa^{\prime}-2}-\kappa+2}, \ldots, s_{M_{N+\kappa^{\prime}-2}}, s_{M_{N+\kappa^{\prime}-2}+1} \text {. }
$$

Therefore, $D_{N+\kappa^{\prime}-2} \subseteq A_{M_{N+\kappa^{\prime}-2}}, \kappa^{\prime}=\kappa, s_{M_{N+i}}=s_{M_{N}+i}$ and $M_{N+i}=M_{N}+i$ for all $0 \leq i \leq \kappa-1$. By induction we get $M_{N+i}=M_{N}+i$ for all $i \in \mathbb{N}_{0}$ as well.

Every salient $p$-point $s_{i} \in \mathfrak{C}$ can be contained in at most two links of $\mathcal{C}_{p}$, and one of them is always the central link of $A_{i}$, which we will denote by $\ell_{p}^{s_{i}}$. Let $K_{s_{i}}$ be the 
arc component of $\ell_{p}^{s_{i}}$ containing $s_{i}$. Given a $p$-point $x \in \mathfrak{C}$ with $L_{p}(x)=l$, there can be two links of $\mathcal{C}_{p}$ containing $x$, but one of them is always $\ell_{p}^{s_{l}}$. We denote the arc component of $\ell_{p}^{s_{l}}$ containing $x$ by $K_{x}$. Let $\ell_{q}^{s_{i}^{\prime}} \in \mathcal{C}_{q}$ and $K_{s_{i}^{\prime}} \subset \ell_{q}^{s_{i}}$ be the similar notation related to $\mathfrak{C}^{\prime}$ and $\mathcal{C}_{q}$. Also, for a $q$-point $x^{\prime}$ of $\mathfrak{C}^{\prime}$ with $L_{q}\left(x^{\prime}\right)=k$ let the arc component of $\ell_{q}^{s^{\prime} k}$ containing $x^{\prime}$ be denoted by $K_{x^{\prime}}$.

Proposition 4.2 There exists $M \in \mathbb{Z}$ such that the following holds:

(1) Let $l \in \mathbb{N}$ and let $x^{\prime}$ be a $q$-point with $L_{q}\left(x^{\prime}\right)=l$. Then $u:=h\left(x^{\prime}\right) \in \ell_{p}^{s_{l}+M}$ and the arc component $K_{u} \subset \ell_{p}^{s_{l}+M}$ containing $u$, also contains a $p$-point $x$ such that $L_{p}(x)=l+M$.

(2) For $l \in \mathbb{N}_{0}$ and $i \in \mathbb{N}$, the number of $q$-points in $\left[s_{i}^{\prime}, s_{i+1}^{\prime}\right]$ with $q$-level $l$ is the same as the number of $p$-points in $\left[s_{M+i}, s_{M+i+1}\right]$ with $p$-level $M+l$.

Proof (1) Recall that the set of $q$-points in $\mathfrak{C}^{\prime}$ is denoted by $E_{q}^{\prime}$. By Theorem 4.1, there exists $M \in \mathbb{Z}$ such that $a_{i}:=h\left(s_{i}^{\prime}\right) \in \ell_{p}^{s_{M+i}}$ for every $i \in \mathbb{N}_{0}$ and the arc component $K_{a_{i}}$ of $\ell_{p}^{s_{M+i}}$ contains $s_{M+i}$. Therefore, statement (1) is true for all salient $q$-points.

Also $h\left(\left[s_{1}^{\prime}, s_{2}^{\prime}\right]\right)=\left[a_{1}, a_{2}\right], s_{M+1} \in K_{a_{1}}$ and $s_{M+2} \in K_{a_{2}}$. Let $q$-point $x_{1}^{\prime} \in\left[s_{2}^{\prime}, s_{3}^{\prime}\right]$ be such that the arc $\left[s_{1}^{\prime}, x_{1}^{\prime}\right]$ is $q$-symmetric with center $s_{2}^{\prime}$. Then $h\left(\left[s_{1}^{\prime}, x_{1}^{\prime}{ }_{1}\right]\right)$ is $p-$ link-symmetric with center $s_{M+2}$. Since there exists a unique $p$-point $b_{1}$ such that the arc $\left[s_{M+1}, b_{1}\right]$ is $p$-symmetric with center $s_{M+2}$, we have $h\left(x_{1}^{\prime}\right) \in K_{b_{1}}$; see Figure 5. Also $L_{q}\left(x_{1}^{\prime}\right)=1$ and $L_{p}\left(b_{1}\right)=M+1$.

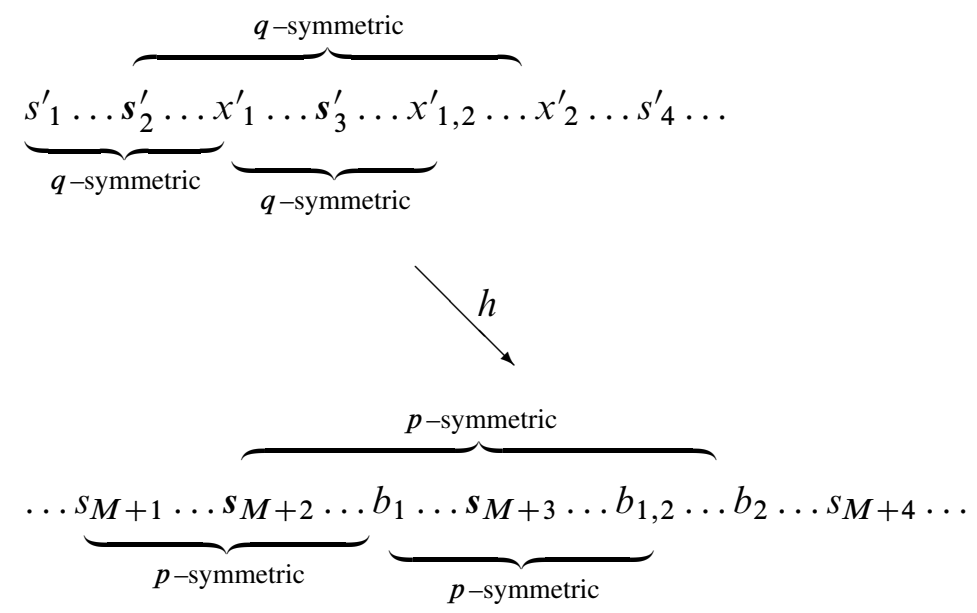

Figure 5: The configuration of symmetric arcs 
We have $h\left(\left[s_{2}^{\prime}, s_{3}^{\prime}\right]\right)=\left[a_{2}, a_{3}\right], s_{M+2} \in K_{a_{2}}$ and $s_{M+3} \in K_{a_{3}}$. Let the $q$-point $x_{2}^{\prime} \in\left[s_{3}^{\prime}, s_{4}^{\prime}\right]$ be such that the arc $\left[s_{2}^{\prime}, x_{2}^{\prime}\right]$ is $q$-symmetric with center $s_{3}^{\prime}$. Therefore $h\left(\left[s_{2}^{\prime}, x_{2}^{\prime}\right]\right)$ is $p$-link-symmetric with center $s_{M+3}$. There exists a unique $p$-point $b_{2}$ such that the arc $\left[s_{M+2}, b_{2}\right]$ is $p$-symmetric with center $s_{M+3}$, so $h\left(x_{2}^{\prime}\right) \in K_{b_{2}}$. Also $L_{q}\left(x_{2}^{\prime}\right)=2$ and $L_{p}\left(b_{2}\right)=M+2$. Since $\left[s^{\prime}{ }_{2}, x_{2}^{\prime}{ }_{2}\right]$ is $q$-symmetric, there exists a $q$-point $x_{1,2}^{\prime} \in\left[s_{3}^{\prime}, x_{2}^{\prime}{ }_{2}\right]$ such that the arc $\left[x_{1}^{\prime}, x_{1,2}^{\prime}\right]$ is $q$-symmetric with center $s^{\prime}{ }_{3}$. Then $h\left(\left[x_{1}^{\prime}, x_{1,2}^{\prime}\right]\right)$ is $p$-link-symmetric with center $s_{M+3}$. Since there exists a unique $p$-point $b_{1,2}$ such that the arc $\left[b_{1}, b_{1,2}\right]$ is $p$-symmetric with center $s_{M+3}$, we have $h\left(x_{1,2}^{\prime}\right) \in K_{b_{1,2}}$; see Figure 5. Also $L_{q}\left(x_{1,2}^{\prime}\right)=1$ and $L_{p}\left(b_{1,2}\right)=M+1$.

The proof of (1) follows by induction. Suppose at step $k$ we have

$$
h\left(\left[s_{k}^{\prime}, s_{k+1}^{\prime}\right]\right)=\left[a_{k}, a_{k+1}\right], \quad s_{M+k} \in K_{a_{k}} \quad \text { and } \quad s_{M+k+1} \in K_{a_{k+1}} ;
$$

see Figure 6. Let again $q$-point $x_{k}^{\prime} \in\left[s^{\prime}{ }_{k+1}, s^{\prime}{ }_{k+2}\right]$ be such that the arc $\left[s_{k}^{\prime}, x_{k}^{\prime}\right]$ is $q$-symmetric with center $s^{\prime}{ }_{k+1}$. Then $h\left(\left[s_{k}^{\prime}{ }_{k}, x_{k}^{\prime}{ }_{k}\right]\right)$ is $p$-link-symmetric with center $s_{M+k+1}$. The unique $p$-point $b_{k}$ such that $\left[s_{M+k}, b_{k}\right]$ is $p$-symmetric with center $s_{M+k+1}$ satisfies $h\left(x_{k}^{\prime}\right) \in K_{b_{k}}$. Also $L_{q}\left(x_{k}^{\prime}\right)=k$ and $L_{p}\left(b_{k}\right)=M+k$.

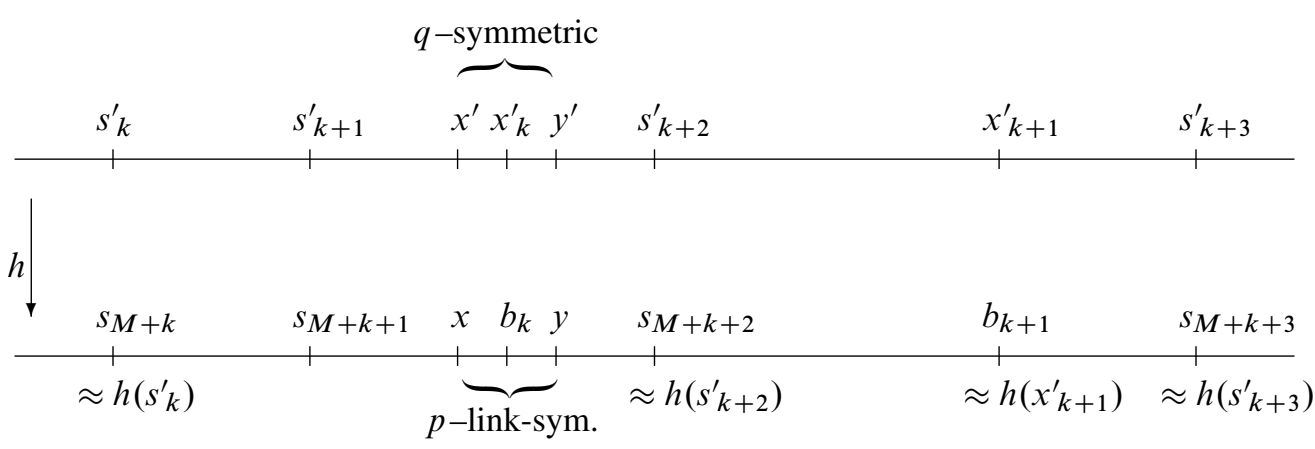

Figure 6: The relative point in the induction step. Here $\approx$ stands for "belongs to the same arc component in the same link".

Let us suppose by induction that for every $q$-point $x^{\prime} \in E_{q}, L_{q}\left(x^{\prime}\right)>0, x^{\prime} \prec x^{\prime}{ }_{k}$, we have $u=h\left(x^{\prime}\right) \in \ell_{p}^{s_{r}+M}$, where $r=L_{q}\left(x^{\prime}\right)$, and the arc component $K_{u} \subset \ell_{p}^{s_{r}+M}$ contains a $p$-point $x$ such that $L_{p}(x)=r+M$. Since $L_{q}\left(x_{k}^{\prime}\right)=k, L_{q}\left(s_{k+1}^{\prime}\right)=k+1$ and $L_{q}\left(s_{k+2}^{\prime}\right)=k+2$, for every $q$-point $x^{\prime} \in\left(s_{k+1}^{\prime}, s_{k+2}^{\prime}\right), x^{\prime} \neq x_{k}^{\prime}$, we have $L_{q}\left(x^{\prime}\right)<L_{q}\left(x_{k}^{\prime}\right)$. Hence for every $q$-point $y^{\prime} \in\left(x_{k}^{\prime}, s^{\prime}{ }_{k+2}\right)$ there exists a $q$-point $x^{\prime} \in\left(s^{\prime}{ }_{k+1}, x_{k}^{\prime}\right)$ such that the arc $\left[x^{\prime}, y^{\prime}\right]$ is $q$-symmetric with center $x_{k}^{\prime}$. So the arc $h\left(\left[x^{\prime}, y^{\prime}\right]\right)$ is $p$-link-symmetric with center $b_{k}$. The induction hypothesis implies that for $u=h\left(x^{\prime}\right)$, the arc component $K_{u} \in \ell_{p}^{s_{r+M}}$ contains a $p$-point $x$ such that $L_{p}(x)=r+M$, where $r=L_{q}\left(x^{\prime}\right)$. 
Since $L_{p}\left(b_{k}\right)=M+k, L_{p}\left(s_{M+k+1}\right)=M+k+1$ and $L_{p}\left(s_{M+k+2}\right)=M+k+2$, we have $L_{p}(v)<L_{p}\left(b_{k}\right)$ for every $p$-point $v \in\left(s_{M+k+1}, s_{M+k+2}\right), v \neq b_{k}$. Hence for every $p$-point $v \in\left(b_{k}, s_{M+k+2}\right)$ there exists a $p$-point $w \in\left(s_{M+k+1}, b_{k}\right)$ such that the arc $[w, v]$ is $p$-symmetric with center $b_{k}$. Therefore, and since $h\left(\left[x^{\prime}, y^{\prime}\right]\right)$ is $p$-link-symmetric with center $b_{k}$, there exists a unique $p$-point $y$ such that the arc $[x, y]$ is $p$-symmetric with center $b_{k}$. Also, $h\left(y^{\prime}\right) \in K_{y}$ and $L_{p}(y)=L_{p}(x)$, so $L_{p}(y)=L_{q}\left(y^{\prime}\right)+M$. This proves that for every $q$-point $x^{\prime} \in E_{q}, L_{q}\left(x^{\prime}\right)>0$, $x^{\prime} \prec s_{k+2}^{\prime}$, we have $u=h\left(x^{\prime}\right) \in \ell_{p}^{s_{r+M}}$, where $r=L_{q}\left(x^{\prime}\right)$, and the arc component $K_{u} \subset \ell_{p}^{s_{r+M}}$ contains a $p$-point $x$ such that $L_{p}(x)=r+M$.

Next $h\left(\left[s_{k+1}^{\prime}, s_{k+2}^{\prime}\right]\right)=\left[a_{k+1}, a_{k+2}\right], s_{M+k+1} \in K_{a_{k+1}}$ and $s_{M+k+2} \in K_{a_{k+2}}$. Let the $q$-point $x_{k+1}^{\prime} \in\left[s_{k+2}^{\prime}, s_{k+3}^{\prime}\right]$ be such that the arc $\left[s_{k+1}^{\prime}, x_{k+1}^{\prime}\right]$ is $q-$ symmetric with center $s^{\prime}{ }_{k+2}$. Then $h\left(\left[s^{\prime}{ }_{k+1}, x_{k+1}^{\prime}\right]\right)$ is $p$-link-symmetric with center $s_{M+k+2}$. Since there exists a unique $p$-point $b_{k+1}$ such that the arc $\left[s_{M+k+1}, b_{k+1}\right]$ is $p$-symmetric with center $s_{M+k+2}$, it follows that $h\left(x_{k+1}^{\prime}\right) \in K_{b_{k+1}}$. Moreover, $L_{q}\left(x_{k+1}^{\prime}\right)=k+1$ and $L_{p}\left(b_{k+1}\right)=M+k+1$. Since $\left[s_{k+1}^{\prime}, x_{k+1}^{\prime}\right]$ is $q$-symmetric with center $s_{k+2}^{\prime}$ and $\left[s_{M+k+1}, b_{k+1}\right]$ is $p$-symmetric with center $s_{M+k+2}$, the same argument as above shows that for every $q$-point $x^{\prime} \in E_{q}, L_{q}\left(x^{\prime}\right)>0, x^{\prime} \prec x^{\prime}{ }_{k+1}$, we have $u=h\left(x^{\prime}\right) \in \ell_{p}^{s_{r}+M}$, where $r=L_{q}\left(x^{\prime}\right)$, and the arc component $K_{u} \subset \ell_{p}^{s_{r}+M}$ contains a $p$-point $x$ such that $L_{p}(x)=r+M$. This proves the induction step.

(2) Let $x$ be a $p$-point such that $L_{p}(x)>0$ and $v=h^{-1}(x)$ lies beyond the $\kappa$-th salient $g$-point. Since $h^{-1}$ is also a homeomorphism and $h^{-1}\left(\mathcal{C}_{p}\right) \prec \mathcal{C}_{g}$, (1) implies that there exists $M^{\prime}$ such that $v \in \ell_{g}^{s^{\prime \prime}} r+M^{\prime}$, where $r=L_{p}(x)$. Also the arc component $K_{v} \subset \ell_{g}^{s^{\prime \prime} r+M^{\prime}}$ contains a $g$-point $x^{\prime \prime}$ such that $L_{g}\left(x^{\prime \prime}\right)=r+M^{\prime}$.

Let $x^{\prime}$ be a $q$-point such that $L_{q}\left(x^{\prime}\right)>0, x^{\prime}$ lies beyond the $\kappa$-th salient $g$-point and $u=h\left(x^{\prime}\right)$ lies beyond the $\kappa$-th salient $p$-point. Then $u \in \ell_{p}{ }^{{ }^{r^{\prime}+M}+M}$, where $r^{\prime}=L_{q}\left(x^{\prime}\right)$, and the arc component $K_{u} \subset \ell_{p}{ }^{s_{r^{\prime}}+M}$ contains a $p$-point $x$ such that $L_{p}(x)=r^{\prime}+M$. Also $v=h^{-1}(x) \in \ell_{g} s^{s^{\prime}+M+M^{\prime}}$ and the arc component $K_{v} \subset \ell_{g} s^{\prime \prime \prime}{ }^{\prime}+M+M^{\prime}$ contains a $g$-point $x^{\prime \prime}$ such that $L_{g}\left(x^{\prime \prime}\right)=L_{q}\left(x^{\prime}\right)+M+M^{\prime}$. Since $h^{-1} \circ h=i d$, we have $x^{\prime \prime}=x^{\prime}$. Also $L_{g}\left(x^{\prime \prime}\right)=L_{q}\left(x^{\prime}\right)+q-g$ implies that $M+M^{\prime}=q-g$. Since the number of $q$-points in $\left[s_{i}^{\prime}, s^{\prime}{ }_{i+1}\right]$ with $q$-level $l, l \in \mathbb{N}_{0}$, is the same as the number of $g$-points in $\left[s^{\prime \prime}{ }_{q-g+i}, s^{\prime \prime}{ }_{q-g+i+1}\right]$ with $g$-level $q-g+l$, it follows that this number is the same as the number of $p$-points in $\left[s_{M+i}, s_{M+i+1}\right]$ with $p$-level $M+l$.

Definition 4.3 A point $x \in \lim \left([0,1], T_{S}\right)$ is called a folding point if it has no closed neighborhood that is homeomorphic to a Cantor set of arcs.

Proof of Theorem 1.1 Folding points $x=\left(\ldots, x_{-2}, x_{-1}, x_{0}\right)$ are characterized by the fact that each entry $x_{-k}$ belongs to the omega-limit set $\omega(c)$ of the turning point $c=\frac{1}{2}$; 
see [28]. If the critical point $c$ of $T_{s}$ has (eventual) period $n$, then $\lim _{\longleftarrow}\left(\left[c_{2}, c_{1}\right], T_{s}\right)$ contains exactly $n$ folding points. If, on the other hand, $\omega(c)$ is infinite, then there are infinitely many folding points.

Since folding points map to folding points under a homeomorphism, $\lim \left([0,1], T_{S}\right)$ and $\lim \left([0,1], T_{S^{\prime}}\right)$ must have the same cardinality of folding points. If this cardinality is finite, then the Ingram conjecture was proved in [31]. So from now on, we can assume that the critical points of $T_{S}$ and $T_{\mathcal{S}^{\prime}}$ have infinite orbits. Therefore the above proposition shows that

$$
F P_{q}\left(\left[s_{k}^{\prime}, s_{k+1}^{\prime}\right]\right)=F P_{p+M}\left(\left[s_{M+k}, s_{M+k+1}\right]\right)=F P_{p}\left(\left[s_{k}, s_{k+1}\right]\right)
$$

for every positive integer $k$, and therefore $F P\left(\mathfrak{C}^{\prime}\right)=F P(\mathfrak{C})$.

Since orbits of $c$ and $c^{\prime}$ are infinite, we have $c_{i}, c^{\prime}{ }_{i} \neq c$ for all $i \in \mathbb{N}$. Note that $c=c^{\prime}=1 / 2, c_{1}, c^{\prime}{ }_{1}>c, c_{2}, c^{\prime}{ }_{2}<c$ and $F P_{p}\left(\left[\alpha, s_{2}\right]\right)=* 0102=F P_{q}\left(\left[\alpha^{\prime}, s^{\prime}{ }_{2}\right]\right)$. It is well-known (see eg [12]) that $s=s^{\prime}$ if and only if $c_{i}$ and $c^{\prime}{ }_{i}$ are on the same side of $c$ for all $i \in \mathbb{N}$ (ie if the kneading sequences of $T_{s}$ and $T_{S^{\prime}}$ are the same). Therefore, we only need to prove that $F P(\mathfrak{C})=F P\left(\mathfrak{C}^{\prime}\right)$ if and only if $c_{i}$ and $c^{\prime}{ }_{i}$ are on the same side of $c$ for all $i \in \mathbb{N}$. We prove this by induction.

Let us suppose that $c_{i}$ and $c^{\prime}{ }_{i}$ are on the same side of $c$ for all $i \leq k$, and that $F P_{p}\left(\left[\alpha, s_{k}\right]\right)=F P_{q}\left(\left[\alpha^{\prime}, s_{k}^{\prime}\right]\right)$. Let us denote by $x$ the first $p$-point on the left of $s_{k}$ (ie $x \in\left[\alpha, s_{k}\right]$ and between $x$ and $s_{k}$ there are no other $p$-points), and let $x^{\prime}$ be the first $q$-point on the left of $s^{\prime}{ }_{k}$. Let $l=L_{p}(x)=L_{q}\left(x^{\prime}\right)$.

If $l=0$, then $L_{p}(\sigma(x))=1=L_{q}\left(\sigma\left(x^{\prime}\right)\right)$. If $c_{k+1}, c^{\prime}{ }_{k+1}<c$, then

$$
F P_{p}\left(\left[\sigma(x), s_{k+1}\right]\right)=F P_{p}\left(\sigma\left(\left[x, s_{k}\right]\right)\right)=10 k+1=F P_{q}\left(\left[\sigma\left(x^{\prime}\right), s_{k+1}^{\prime}\right]\right) .
$$

If $c_{k+1}, c^{\prime}{ }_{k+1}>c$, then

$$
F P_{p}\left(\left[\sigma(x), s_{k+1}\right]\right)=1 k+1=F P_{q}\left(\left[\sigma\left(x^{\prime}\right), s_{k+1}^{\prime}\right]\right) .
$$

Similarly $c_{k+1}<c$ and $c_{k+1}^{\prime}>c$, or vice versa, implies

$$
F P_{p}\left(\left[\sigma(x), s_{k+1}\right]\right) \neq F P_{q}\left(\left[\sigma\left(x^{\prime}\right), s_{k+1}^{\prime}\right]\right)
$$

If $l \neq 0$, then since $x \in\left(s_{k-1}, s_{k}\right)$, we have $l<k-1$, ie $l+1<k$, and by the induction hypothesis $c_{l+1}$ and $c^{\prime}{ }_{l+1}$ are on the same side of $c$. Therefore, if $c_{k+1}$ and $c^{\prime}{ }_{k+1}$ are on the same side of $c$ as $c_{l+1}$, then

$$
F P_{p}\left(\left[\sigma(x), s_{k+1}\right]\right)=l+1 \quad k+1=F P_{q}\left(\left[\sigma\left(x^{\prime}\right), s_{k+1}^{\prime}\right]\right) .
$$


If $c_{k+1}$ and $c^{\prime}{ }_{k+1}$ are on the same side of $c$, but on the opposite side of $c_{l+1}$, then

$$
F P_{p}\left(\left[\sigma(x), s_{k+1}\right]\right)=l+10 k+1=F P_{q}\left(\left[\sigma\left(x^{\prime}\right), s_{k+1}^{\prime}\right]\right) .
$$

Similarly, $c_{k+1}<c$ and $c_{k+1}^{\prime}>c$, or vice versa, implies

$$
F P_{p}\left(\left[\sigma(x), s_{k+1}\right]\right) \neq F P_{q}\left(\left[\sigma\left(x^{\prime}\right), s_{k+1}^{\prime}\right]\right) .
$$

In every case we conclude that $c_{i}$ and $c^{\prime}{ }_{i}$ are on the same side of $c$ for all $i \leq k+1$ if and only if $F P_{p}\left(\left[\alpha, s_{k+1}\right]\right)=F P_{q}\left(\left[\alpha^{\prime}, s^{\prime}{ }_{k+1}\right]\right)$, and by induction that $c_{i}$ and $c^{\prime}{ }_{i}$ are on the same side of $c$ for all $i \in \mathbb{N}$ if and only if $F P(\mathfrak{C})=F P\left(\mathfrak{C}^{\prime}\right)$.

This proves the Ingram conjecture.

\section{Pseudo-isotopy}

Throughout this section, $h: \lim \left([0,1], T_{s}\right) \rightarrow \underset{\lim }{\longleftarrow}\left([0,1], T_{s}\right)$ will be an arbitrary self-

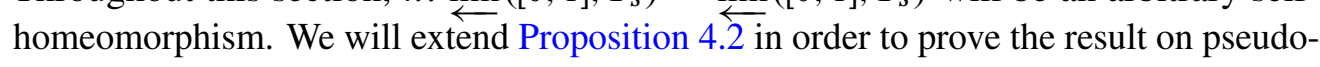
isotopy. Note that (1) and (2) of Proposition 4.2 together show that $h$ induces an order preserving injection $h_{q, p}$ from $E_{q}$ to $E_{p}$ such that $h_{q, p}\left(E_{q, i}\right)=E_{p, M+i}=E_{p+M, i}$ for every $i \in \mathbb{N}_{0}$, where $E_{r, l}$ denotes the set of all $r$-points with $r$-level $l$ (see Definition 2.5). In fact $h_{q, p}$ is an order preserving bijection from $E_{q}$ to $E_{p+M}$ and is defined as follows:

Definition 5.1 Let $x \in E_{q}$. If $x=s_{i}^{\prime}$ for some $i \in \mathbb{N}$, we define $h_{q, p}\left(s_{i}^{\prime}\right)=$ $s_{M+i} \in E_{p}$. For all other $x \in E_{q}$, there exists $i \in \mathbb{N}$ such that $x \in\left(s_{i}^{\prime}, s_{i+1}^{\prime}\right)$. By Proposition 4.2, the number of $q$-points of $\left(s_{i}^{\prime}, s_{i+1}^{\prime}\right)$ is the same as the number of $(p+M)$-points of $\left(s_{M+i}, s^{\prime}{ }_{M+i+1}\right)$. Let $\left(s_{i}^{\prime}, s_{i+1}^{\prime}\right) \cap E_{q}=\left\{x^{0}, \ldots, x^{n}\right\}$ and $\left(s_{M+i}, s_{M+i+1}^{\prime}\right) \cap E_{p+M}=\left\{y^{0}, \ldots, y^{n}\right\}$. We define $h_{q, p}\left(x^{i}\right)=y^{i}, i=0, \ldots, n$.

The next lemma shows that $h_{q, p}$ is essentially independent of $q$ and $p$.

Lemma 5.2 If $q_{1}, p_{1} \in \mathbb{N}$ are such that $h\left(\mathcal{C}_{q_{1}}\right) \prec \mathcal{C}_{p_{1}} \prec h\left(\mathcal{C}_{q}\right) \prec \mathcal{C}_{p}$, then

$$
\left.h_{q_{1}, p_{1}}\right|_{E_{q_{1}}}=\left.h_{q, p}\right|_{E_{q_{1}}} .
$$

Proof By Proposition 4.2, $h\left(\mathcal{C}_{q}\right) \prec \mathcal{C}_{p}$ implies that there exists $M \in \mathbb{Z}$ such that $h_{q, p}\left(E_{q, i}\right)=E_{p, M+i}$ for every $i \in \mathbb{N}_{0}$. Also, $h\left(\mathcal{C}_{q_{1}}\right) \prec \mathcal{C}_{p_{1}}$ implies that there exists $M_{1} \in \mathbb{Z}$ such that $h_{q_{1}, p_{1}}\left(E_{q_{1}, i}\right)=E_{p_{1}, M_{1}+i}$ for every $i \in \mathbb{N}_{0}$. Let $r, l \in \mathbb{N}$ be such that $q_{1}=q+r$ and $p_{1}=p+l$. Since $E_{q+r, i}=E_{q, r+i}$, we have

$$
h_{q, p}\left(E_{q+r, i}\right)=h_{q, p}\left(E_{q, r+i}\right)=E_{p, M+r+i},
$$


and also

$$
h_{q+r, p+l}\left(E_{q+r, i}\right)=E_{p+l, M_{1}+i}=E_{p, M_{1}+l+i} .
$$

We want to prove that $M+r=M_{1}+l$. To see this it suffices to pick a convenient point $x$ in $E_{q+r, j}$ for some $j \in \mathbb{N}$, and to prove that $h_{q, p}(x)=y=h_{q+r, p+l}(x)$. Then the fact that $y \in E_{p, M+r+j}$ and $y \in E_{p, M_{1}+l+j}$ implies that $M+r+j=L_{p}(y)=M_{1}+l+j$. For us, the convenient choice of $x \in E_{q+r} \subset E_{q}$ is a salient $(q+r)$-point.

Let us denote the salient $(q+r)$-points by $\hat{s}_{i}^{\prime}$ and the salient $(p+l)$-points by $\widehat{s}_{i}$, while as before $s_{i}^{\prime}$ denotes the salient $q$-points and $s_{i}$ denotes the salient $p$-points. Note that the salient $(q+r)$-point $\hat{s}_{i}^{\prime}$ is the same as the salient $q$-point $s_{i+r}^{\prime}$, and the salient $(p+l)$-point $\widehat{s}_{i}$ is the same as the salient $p$-point $s_{i+l}$. Let us denote the maximal $(q+r)$-link-symmetric arc with the center $\widehat{S}_{i}^{\prime}$ by $\widehat{A}_{i}^{\prime}$, and the maximal $(p+l)$-link-symmetric arc with the center $\widehat{s}_{i}$ by $\hat{A}_{i}$, while as before $A_{i}^{\prime}$ denotes the maximal $q$-link-symmetric arc with the center $s_{i}^{\prime}$, and $A_{i}$ denotes the maximal $p$-linksymmetric arc with the center $s_{i}$. Note that $h\left(\hat{A}_{i}^{\prime}\right) \subseteq \widehat{A}_{M_{1}+i}, h\left(A_{i+r}^{\prime}\right) \subseteq A_{M+i+r}$ and $\widehat{s}_{i}^{\prime}=s_{i+r}^{\prime}$. Also, the center of $\hat{A}_{M_{1}+i}$ is $\widehat{s}_{M_{1}+i}=s_{M_{1}+i+l}$ and the center of $A_{M+i+r}$ is $s_{M+i+r}$. Therefore, $s_{M+i+r}=s_{M_{1}+i+l}$ and $M+r=M_{1}+l$.

Corollary $5.3 R=M+p-q$ does not depend on $M, p, q$.

Proof By Lemma 5.2, $M_{1}+l=M+r$. Therefore

$$
R_{1}=M_{1}+p_{1}-q_{1}=M_{1}+(p+l)-(q+r)=M+r+p-q-r=R .
$$

Definition 5.4 We call an arc $B \subset \mathfrak{C}$ a $p$-bridge if the boundary points of $B$ are $p$-points with $p$-level 0 , and if $L_{p}(x) \neq 0$ for every $p$-point $x \in \operatorname{Int} B$.

Corollary 5.5 Let $B^{\prime} \subset \mathfrak{C}$ be a $(q+1)$-bridge and $\partial B^{\prime}=\left\{a^{\prime}, b^{\prime}\right\}$. There exists a $(p+M+1)$-bridge $B$ such that for $\partial B=\{a, b\}$ we have $h\left(B^{\prime}\right) \subset K_{a} \cup B \cup K_{b}$ and $h\left(a^{\prime}\right) \in K_{a}, h\left(b^{\prime}\right) \in K_{b}$, where $K_{a}$ and $K_{b}$ are the arc-components of the link $\ell_{p}^{s_{M+1}}$ of $\mathcal{C}_{p}$ containing $a$ and $b$ respectively.

Proof Proposition 4.2 dealt with points in $E_{q, j}$ for $j \geq 1$, but bridges involve points of level zero. Since $E_{q, 1}=E_{q+1,0}$, in this corollary we can work with $(q+1)$-bridges.

For each $j \geq 1, E_{q, j}$ is contained in a single link $\ell_{q} s_{j}^{\prime} \in \mathcal{C}_{q}$ and by Proposition 4.2, for $\ell_{p}^{s_{M+j}} \supseteq h\left(\ell_{q}^{s_{j}^{\prime}}\right)$, every point of $h\left(E_{q, j}\right)$ is contained in an arc component of $\ell_{p}^{s_{M+j}}$ which contains a $p$-point of $E_{p, M+j}=E_{p+M, j}$. Since $E_{q+1,0}=E_{q, 1}$ and $E_{p+M+1,0}=E_{p+M, 1}$, every point of $h\left(E_{q+1,0}\right)=h\left(E_{q, 1}\right)$ is contained in an arc component of $\ell_{p}^{s_{M+1}}$ which contains a point of $E_{p+M, 1}=E_{p+M+1,0}$. 
Every two adjacent points of $E_{q+1,0}$ are the boundary points of a $(q+1)$-bridge, and every two adjacent points of $E_{p+M+1,0}$ are the boundary points of a $(p+M+1)-$ bridge. We also have $h_{q, p+M}\left(E_{q+1,0}\right)=h_{q, p+M}\left(E_{q, 1}\right)=E_{p, M+1}=E_{p+M+1,0}$. Therefore, for every $(q+1)$-bridge $B^{\prime}$ there exists a $(p+M+1)$-bridge $B$ such that $h_{q, p+M}\left(B^{\prime}\right)=B$. More precisely, for every $(q+1)$-bridge $B^{\prime}$ and $\partial B^{\prime}=\left\{a^{\prime}, b^{\prime}\right\}$, there exists a $(p+M+1)$-bridge $B$ such that for $\partial B=\{a, b\}$ we have $h\left(B^{\prime}\right) \subset$ $K_{a} \cup B \cup K_{b}$ with $h\left(a^{\prime}\right) \in K_{a}$ and $h\left(b^{\prime}\right) \in K_{b}$. Note that if $B^{\prime}$ is a $(q+1)$-bridge with center $z^{\prime}$ and $\partial B^{\prime}=\left\{a^{\prime}, b^{\prime}\right\}$ and $B^{\prime}$ is contained in a single link $\ell_{q+1} s^{\prime}{ }_{1}$, then $h\left(B^{\prime}\right)$ is contained in the arc component $K_{a}=K_{b}$ which contains also a $(p+M+1)-$ point $z$ such that $L_{p+M+1}(z)=L_{q+1}\left(z^{\prime}\right)$. So the arc component $K_{a}$ contains a $(p+M+1)$-bridge $B$ with center $z$ and we have again $h\left(B^{\prime}\right) \subset K_{a} \cup B \cup K_{b}$.

Example 5.6 A $\sin \frac{1}{x}$-continuum is a homeomorphic copy of

$$
(\{0\} \times[-1,1]) \cup\left\{\left(x, \sin \frac{1}{x}\right): x \in(0,1]\right\}
$$

and the arc $\{0\} \times[-1,1]$ is called the bar of the $\sin \frac{1}{x}-$ continuum. Assume that $s>\sqrt{2}$ is such that the inverse limit $\lim \left([0,1], T_{S}\right)$ contains a $\sin \frac{1}{x}$-continuum $H$. (Such $s$ exist in abundance; see [2] and [15].) Then $\left\{\sigma^{-n}(H)\right\}_{n=0}^{\infty}$ is a sequence of pairwise disjoint $\sin \frac{1}{x}$-continua with $\operatorname{diam}\left(\sigma^{-n}(H)\right) \rightarrow 0$ as $n \rightarrow \infty$. There is then a sequence of disjoint neighborhoods $U_{n}$ of $\sigma^{-n}(H)$ with $\operatorname{diam}\left(U_{n}\right) \rightarrow 0$. For each $n, U_{n} \cap \mathfrak{C}$ contains arbitrarily long arcs. Pick a sequence of arcs $A_{n} \subset U_{n} \cap \mathfrak{C}$ of arc-length $\geq n+1$, and construct a bijection $h: \lim \left([0,1], T_{S}\right) \circlearrowleft$ such that $h$ is the identity on $\lim \left([0,1], T_{s}\right) \backslash \bigcup_{n} A_{n}$ and on each $A_{n}, h$ fixes $\partial A_{n}$, but moves some points in $\overleftarrow{A_{n}}$ homeomorphically such that there is $x_{n} \in A_{n}$ with $\bar{d}\left(x_{n}, h\left(x_{n}\right)\right)=n$. Since $\operatorname{diam}\left(U_{n}\right) \rightarrow 0$, we find that $h$ is continuous and bijective. Finally the compactness of $\lim \left([0,1], T_{S}\right)$ implies that $h$ is a homeomorphism. Even though $h$ is isotopic to the

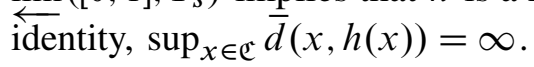

Therefore we cannot assume that a general self-homeomorphism of $\underset{\lim }{\longleftarrow}\left([0,1], T_{S}\right)$ has an $R \in \mathbb{Z}$ such that $\sup _{x} \bar{d}\left(h(x), \sigma^{R}(x)\right)<\infty$. Block, Jakimovik and Keesling [9, Theorem 4.2] used this property to conclude that $h$ and $\sigma^{R}$ are pseudo-isotopic, ie they permute the composants of $\lim \left(\left[c_{2}, c_{1}\right], T_{S}\right)$ in the same way. However, since $\sigma^{-R} \circ h$ preserves $(q+1)$-bridges for some $R \in \mathbb{Z}$ and $q$ sufficiently large, we can still follow the argument from [9].

Proof of Theorem 1.3 Let $P=s /(1+s)>1 / 2$ be the orientation reversing fixed point of $T_{s}$ and $Q$ the center between $c_{2}$ and $c_{1}$. Let $\varepsilon=\operatorname{mesh}\left(\mathcal{C}_{p}\right)$ in Definition 2.4. Without loss of generality, we can take $\varepsilon / 2<\min \{|c-P|,|c-Q|\}$. Let

$$
x \in \lim _{\longleftarrow}\left([0,1], T_{s}\right) \backslash \mathfrak{C}=\lim _{\longleftarrow}\left(\left[c_{2}, c_{1}\right], T_{s}\right)
$$


be arbitrary. Recall that the composant of $x$ in $\lim _{\longleftarrow}\left(\left[c_{2}, c_{1}\right], T_{s}\right)$ is the union of all proper subcontinua of $\lim \left(\left[c_{2}, c_{1}\right], T_{s}\right)$ containing $\overleftarrow{x}$. Without loss of generality we can fix $q \in \mathbb{N}$ such that $\overleftarrow{\pi}_{q+1}(x) \geq P$. Fix $p \in \mathbb{N}$ and $M \in \mathbb{Z}$ as in Proposition 4.2 such that $h\left(\mathcal{C}_{q}\right) \preceq \mathcal{C}_{p}$ and $h$ sends $(q+1)$-bridges to $(p+M+1)$-bridges in terms of Corollary 5.5. Let $R=M+p-q$, so $p+M+1=q+R+1$. Since by Corollary 5.3, $R$ does not depend on $q$ and $p$, we can take $q$ and $p$ larger than $|R|$ without loss of generality.

Recall that the links $\ell_{p}{ }^{k}$ of $\mathcal{C}_{p}$ are of the form $\ell_{p}{ }^{k}=\pi_{p}^{-1}\left(I_{p}{ }^{k}\right)$ of width $\leq \varepsilon s^{-p} / 2$. The map $\sigma^{-R}$ maps the chain $\mathcal{C}_{p}$ to a chain $\tilde{\mathcal{C}}_{p-R}$ whose links are of the form $\pi_{p-R}^{-1}\left(I_{p}{ }^{k}\right)$ and hence also with width $\leq \varepsilon s^{-p} / 2$; this chain is coarser than $\mathcal{C}_{p-R}$ if $R<0$. Furthermore, the $\sigma^{-R}$-image of a $(q+R+1)$-bridge is a $(q+1)$-bridge.

Take $\tilde{h}=\sigma^{-R} \circ h$. Since $h\left(\mathcal{C}_{q}\right) \preceq \mathcal{C}_{p}$, we have $\tilde{h}\left(\mathcal{C}_{q}\right) \preceq \widetilde{\mathcal{C}}_{p-R}$ and $\tilde{h}$ sends $(q+1)-$ bridges to $(q+1)$-bridges, but the "error" allowed in Corollary 5.5, ie the arccomponents of links from $\mathcal{C}_{p}$, must now be replaced by arc-components of links of $\widetilde{\mathcal{C}}_{p-R}$. Recall that width $\left(\mathcal{C}_{p}\right)=\max _{j}\left|I_{p}^{j}\right|$, and $\left|\pi_{p-i}\left(\ell_{p}^{j}\right)\right|=\left|\pi_{p}\left(\ell_{p}^{j}\right)\right| s^{i}=\left|I_{p}{ }^{j}\right| s^{i}$, for every $0 \leq i \leq p$. Therefore,

$$
\pi_{p-R}\left(\tilde{\ell}_{p-R}^{j}\right)=\pi_{q-M}\left(\tilde{\ell}_{q-M}^{j}\right) \leq \varepsilon s^{-p} / 2
$$

and

$$
\pi_{q+1}\left(\tilde{\ell}_{p-R}^{j}\right)=\pi_{q+1}\left(\tilde{\ell}_{q-M}^{j}\right)=\pi_{q-M}\left(\tilde{\ell}_{q-M}^{j}\right) s^{-M-1} \leq \varepsilon s^{-p-M-1} / 2 .
$$

Thus, the $(q+1)-$ th projection of links of $\widetilde{\mathcal{C}}_{p-R}$ are intervals and their lengths are $\leq \varepsilon s^{-(p+M+1)} / 2=\varepsilon s^{-(q+R+1)} / 2$; see Figure 7.

The $(q+1)$-bridges that are small enough to belong to one or two links of $\mathcal{C}_{q}$ will map to arcs contained in the link $\tilde{\ell}_{p-R}$. Since $\pi_{q+1}(x) \geq P$ and $\varepsilon s^{-(q+1)} / 2<|c-P|$, no such short bridge can be close to $x$. On the longer $(q+1)$-bridges of $\mathcal{C}_{q}$ that map outside of $\tilde{\ell}_{p-R}, \widetilde{h}$ acts as a trivial one-to-one correspondence, sending the first such bridge to the first, the second to the second, etc.

Find a sequence $\left(x_{n}\right)_{n \in \mathbb{N}} \subset \mathfrak{C}$ such that $x_{n} \rightarrow x$. Then for large $n, x_{n}$ belongs to a long $(q+1)$-bridge, and by the above argument, $\tilde{h}\left(x_{n}\right)$ and $x_{n}$ belong to the same $(q+1)$-bridge up to an "error" of at most $\varepsilon s^{-(q+R+1)} / 2$. Take $H_{n}=\left[\tilde{h}\left(x_{n}\right), x_{n}\right]$ and a subsequence such that $H_{n_{j}} \rightarrow H$ in Hausdorff topology. Clearly $H$ is a continuum and $x, \tilde{h}(x) \in H$. Since $\pi_{q+1}(x) \geq P$, the arcs $H_{n_{j}}$ belong to arcs whose $(q+1)$-projections belong to $\left[c-\varepsilon s^{-(q+R+1)} / 2, c_{1}\right]$ for all sufficiently large $j$. Since $q+R+1 \geq 1$ and $\varepsilon / 2<c-Q$, we have $Q<c-\varepsilon / 2<c-\varepsilon s^{-(q+R+1)} / 2$ implying $\left[c-\varepsilon S^{-(q+R+1)} / 2, c_{1}\right] \subset\left[Q, c_{1}\right]$. 

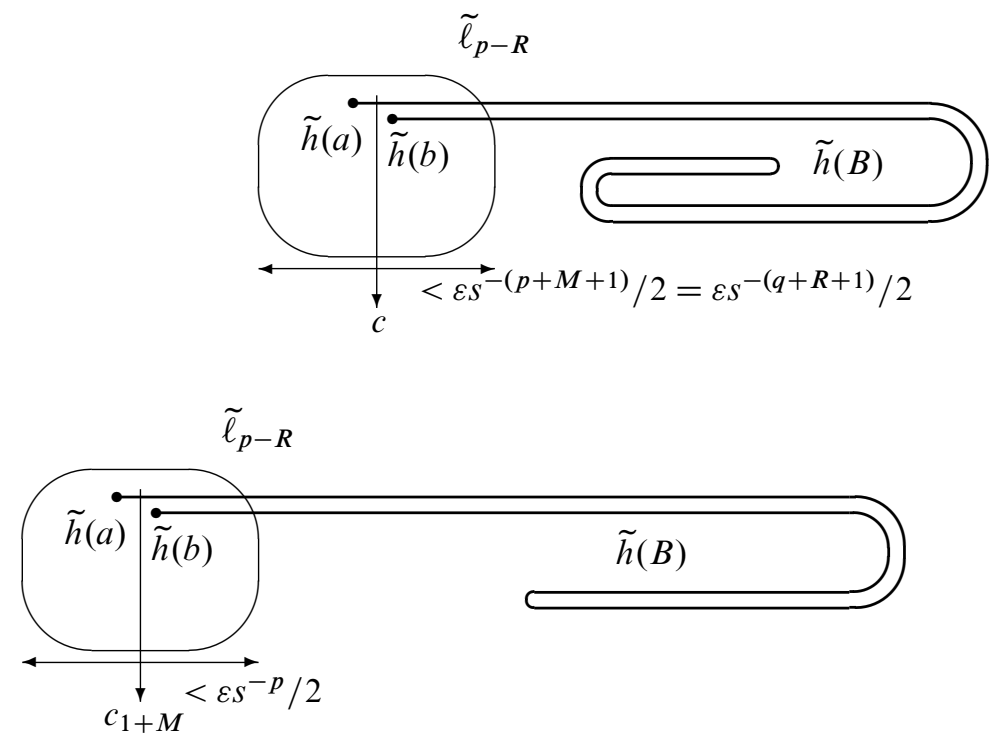

Figure 7: The $(p-R)$-th and $(q+1)$-th projection of "the bridge" $\widetilde{h}(B)$ with relevant link $\tilde{\ell}_{p-R}$. The picture is suggestive of $M+1 \leq 0$; if instead $M+1>0$, then $\tilde{h}(B)$ contains fewer $(q+1)$-points than $(p-R)$-points.

Therefore $\pi_{q+1}\left(H_{n_{j}}\right), \pi_{q+1}(H) \subset\left[Q, c_{1}\right]$, and since $\left[Q, c_{1}\right]$ is a proper subset of $\left[c_{2}, c_{1}\right]$ and the inclusion holds for arbitrarily large $q, H$ is a proper subcontinuum of $\lim \left(\left[c_{2}, c_{1}\right], T_{s}\right)$. It follows that $\tilde{h}(x)$ and $x$ belong to the same composant of

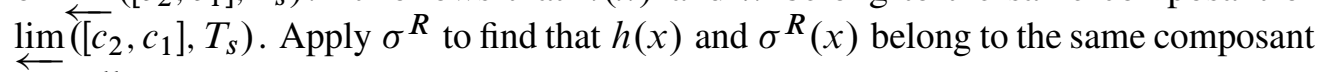
as well.

Pseudo-isotopy of $h$ implies that the number of composants being mapped to themselves is the same for $h^{n}$ and $\sigma^{n R}$. This number grows like $s^{n R}$, which in [8] provides a proof of the Ingram conjecture for tent maps with periodic critical point. In this situation, [8] in fact also shows that $h$ is isotopic to a power of the shift. Due to the existence of composants that are not arc-connected, this is not so clear in the general case.

Remark 5.7 Not every pseudo-isotopy is an isotopy. For instance, a homeomorphism flipping the bar of a $\sin \frac{1}{x}$-continuum cannot be isotopic to the identity. If the bonding map is a quadratic map within the first period doubling cascade, then the inverse limit space is a finite collection of $\sin \frac{1}{x}$-continua (see [7]) and we can indeed construct homeomorphism that are pseudo-isotopic but not isotopic to the identity. Among those tent maps $T_{s}, s \in[\sqrt{2}, 2]$, whose inverse limit space is known to contain $\sin \frac{1}{x}-$ continua, both in [2] and [15], the topology is much more complicated, as more than a 
single ray can be expected to accumulate on their bars. Thus the following question is very relevant:

Is every self-homeomorphism of $\underset{\longleftarrow}{\lim }\left([0,1], T_{S}\right)$ isotopic to a power of the shift?

We know this to be true if $c$ is periodic or nonrecurrent [8;10], but this case is simpler, because the only proper subcontinua of $\lim _{\longleftarrow}\left(\left[c_{2}, c_{1}\right], T_{s}\right)$ are arcs or points.

\section{The logistic family}

In this section we prove of Theorem 1.2. Logistic maps are by far the best studied unimodal maps, and we give here a list of its properties that simplify our task, although they are not essential: Theorem 1.2 works for arbitrary $C^{2}$ families just as well.

The map $f_{a}(x)=a x(1-x)$ has negative Schwarzian derivative, ie

$$
S f_{a}:=\frac{f_{a}^{\prime \prime \prime}}{f_{a}^{\prime}}-\frac{3}{2}\left(\frac{f_{a}^{\prime \prime}}{f_{a}^{\prime}}\right)^{2}<0
$$

wherever defined. Due to Singer [30], this implies that every $p$-periodic point $x$ with multiplier $\left|\left(f_{a}^{p}\right)^{\prime}(x)\right| \leq 1$ is attracting from at least one side, and has the critical point in its immediate basin of its orbit, ie there is a point $y \in \operatorname{orb}(x)$ such that $\bigcap_{k} f_{a}^{p k}([y, c])=\{y\}$. Another consequence of the Schwarzian derivative being negative (Guckenheimer [19]) is that every interval $K$ on which $\left.f^{n}\right|_{K}$ is a homeomorphism for all $n \geq 0$ must be attracted by a periodic attractor, so there are no wandering intervals. The absence of wandering intervals was proved for general $C^{2}$ families; see de Melo and van Strien [24, Theorem 6.2. page 156].

Much more complicated results have been proved for the logistic family as well. The entropy $h_{\text {top }}\left(f_{a}\right)$ is a nondecreasing function of its parameter; see Douady [16] and Milnor and Thurston [25]. Furthermore, if $f_{a}$ and $f_{b}$ are topological conjugate and have no periodic attractor, then $a=b$. This is known as "denseness of hyperbolicity" and was proved by Graczyk and Światek [18] and Lyubich [23].

The logistic family $\left(f_{a}\right)_{a \in[0,4]}$ is richer than the tent family $\left(T_{s}\right)_{s \in[0,2]}$ in the sense that it allows renormalization of all types.

Definition 6.1 A unimodal map $f:[0,1] \rightarrow[0,1]$ with critical point $c$ is renormalizable if there is a neighborhood $J \ni c$ and period $p \geq 2$ such that

$$
\begin{aligned}
& f^{p}(J) \subset J, \quad f^{p}(\partial J) \subset \partial J \text { and } \\
& J, f(J), \ldots, f^{p-1}(J) \text { have disjoint interiors. }
\end{aligned}
$$

In this case, $f^{p}: J \rightarrow J$ is a new unimodal map; it is called a renormalization of $f$. 
If $f_{a}$ is renormalizable, then $\left.f_{a}^{p}\right|_{J}$ is conjugate with another member, $f_{a^{\prime}}$, of the logistic family. This is because the logistic family is full in the sense that it witnesses every possible combinatorial type of unimodal map without wandering intervals, [24, Section II.4]. The renormalization of a unimodal map can itself be renormalizable, etc. This gives rise to infinitely renormalizable maps, for which there is a nested sequence $\left(J^{k}\right)_{k \geq 1}$ of $p_{k}$-periodic neighborhoods of $c$, such that $C:=\bigcap_{k} \bigcup_{j=0}^{p_{k}-1} f^{j}\left(J^{k}\right)$ is an invariant Cantor set, called a solenoidal attractor. It coincides with $\omega(c)$, is Lyapunov stable and the orbit of Lebesgue-a.e. point converges to $C$; yet arbitrarily close to $C$ there are periodic orbits that are not contained in $C$.

Definition 6.2 We call a point $x$ nonwandering if for every neighborhood $U \ni x$, there is $n \geq 1$ such that $f^{n}(U) \cap U \neq \varnothing$. The collection $\Omega(f)$ of nonwandering points is called the nonwandering set. The reduced nonwandering set of a logistic map $f_{a}, \Omega\left(f_{a}\right) / \sim_{s n}$, is the nonwandering set of $f_{a}$ with each saddle-node pair identified to a point. This set inherits an order from $[0,1]$, and we say that two logistic maps $f_{a}$ and $f_{b}:[0,1] \rightarrow[0,1]$ are order-preserving conjugate on their reduced nonwandering sets if there is an order preserving homeomorphism $h: \Omega\left(f_{a}\right) / \sim_{s n} \rightarrow \Omega\left(f_{b}\right) / \sim_{s n}$ such that $f_{b} \circ h([x])=h \circ f_{a}([x])$ for all $[x] \in \Omega\left(f_{a}\right) / \sim_{s n}$.

Clearly, periodic points are nonwandering, as are points in the closure of the set of periodic points. For logistic maps, the only wandering points are those that belong to the basin of a periodic attractor (but are not periodic themselves), or that belong to the basin of a solenoidal attractor.

Let us describe in some detail the first periodic doubling cascade, occurring at parameters $1=a_{0}<a_{1}<a_{2}<\ldots$ on the parameter interval $\left[0, a_{\infty}\right]$ (see Figure 8), where $a_{\infty}=\lim _{k} a_{k}$ is the so-called Feigenbaum-Coullet-Tresser parameter [17; 34], at which $f_{a}$ has the simplest kind of solenoidal attractor. The map $f_{a_{\infty}}$ is also called the $2^{\infty}$-map, because it has periodic points of period $2^{k}$ for all $k$, and none of higher period in the Sharkovskiu order. These dynamics are not present in the tent family. It is also well-known that $h_{\text {top }}\left(f_{a}\right)=0$ if and only if $a \leq a_{\infty}$; see [27]. The inverse limits spaces $\lim _{\longleftarrow}\left([0,1], f_{a}\right)$ for $a \in\left[0, a_{\infty}\right]$ were described in [7].

- For $a \in\left[0, a_{0}\right], f_{a}$ has a single fixed point 0 , attracting every $x \in[0,1]$. The inverse limit $\lim _{\longleftarrow}\left([0,1], f_{a}\right)$ is a single point $(\ldots, 0,0,0)$.

- At $a=a_{0}$, the fixed point 0 becomes unstable and splits off another stable fixed point $p_{a}=(a-1) / a$ with multiplier $f^{\prime}{ }_{a}\left(p_{a}\right)=2-a$, attracting every point $x \in(0,1)$. For all $a \in\left(a_{0}, a_{1}=3\right], p_{a}$ remains stable and $\underset{\leftarrow}{\lim }\left([0,1], f_{a}\right)$ is a single arc. 


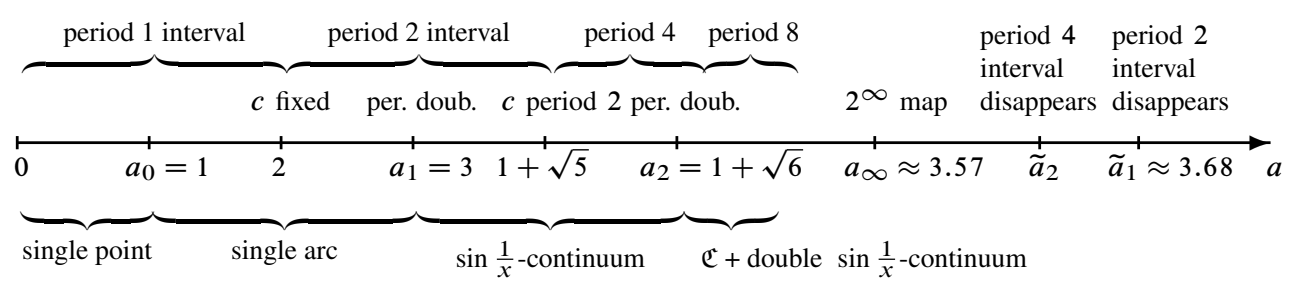

Figure 8: Sketch (not to scale) of the bifurcations in the first period doubling cascade. The upper braces indicate parameter intervals in which $J^{k}$ is the smallest periodic interval as in (6-1). The lower braces indicate parameter intervals where the inverse limit space is as indicated.

- At $a=a_{1}, p_{a}$ undergoes a period doubling bifurcation. It becomes unstable itself, and splits off a stable period 2 orbit

$$
q_{a}^{ \pm}=\frac{a+1 \pm \sqrt{(a-1)^{2}-4}}{2 a}
$$

with multiplier $\left(f_{a}^{2}\right)^{\prime}\left(q_{a}^{ \pm}\right)=5-(a-1)^{2}$. For all $a \in\left(a_{1}, a_{2}=1+\sqrt{6}\right], q_{a}^{ \pm}$remain stable and $\lim _{\longleftarrow}\left([0,1], f_{a}\right)$ is a $\sin \frac{1}{x}$-continuum, consisting of two arc components: the zero-composant and the bar (an arc containing $\left(\ldots, p_{a}, p_{a}, p_{a}\right)$ ).

- At $a=a_{2}, q_{a}^{ \pm}$undergo a period doubling bifurcation. They become unstable themselves, splitting off a stable period 4 orbit. For all $a \in\left(a_{2}, a_{3}\right]$, this period 4 orbit remains stable and $\lim \left([0,1], f_{a}\right)$ has four arc-components: two arcs containing $\left(\ldots, q_{a}^{-}, q_{a}^{+}, q_{a}^{-}, q_{a}^{+}\right) \overleftarrow{\text { and }}\left(\ldots, q_{a}^{+}, q_{a}^{-}, q_{a}^{+}, q_{a}^{-}\right)$respectively, one ray containing $\left(\ldots, p_{a}, p_{a}, p_{a}\right)$ and forming a double sin $\frac{1}{x}$-continuum with the two arcs, and the zero-composant compactifying on all of the above.

- In general, for $a \in\left(a_{k}, a_{k+1}\right], k \geq 1$, the inverse limit space $\lim _{\longleftarrow}\left([0,1], f_{a}\right)$ has $2^{k}$ arc-components arranged in a hierarchical manner. There are $2^{k-1}$ arcs, each containing a $2^{k-1}$-periodic point of the shift homeomorphism. At the next level, there are $2^{k-2}$ rays, each containing a $2^{k-2}$-periodic point and forming a double $\sin \frac{1}{x}-$ continuum with two arcs of the first level. The next level consists of $2^{k-2}$ rays, each containing a $2^{k-2}$-periodic point, and compactifying on two double $\sin \frac{1}{x}$-continua (including four arcs) of the previous levels. This structure continues, until, at the highest level, the zero-composant compactifies on all of the above.

- At $a=a_{\infty}$, this structure contains infinitely many levels, and compactifies on an additional Cantor set of points $x$ such that $x_{-n} \in \omega(c)$ for all $n \geq 0$.

We see that the inverse limit $\lim _{\longleftarrow}\left([0,1], f_{a}\right)$ is different at every stage of the period doubling cascade. 
Each saddle node bifurcation occurring at $a_{*}>a_{\infty}$, is followed by its own period doubling cascade. At $a=a_{*}$, a fresh neutrally attracting $p$-periodic orbit emerges, which immediately splits into an unstable and a stable $p$-periodic orbit, but this does not affect the inverse limit space. For this reason we introduced the reduced nonwandering set which does not discriminate between the twin $p$-periodic orbits emerging from a saddle node bifurcation. The nonwandering sets $\Omega\left(f_{a_{*}}\right)$ and $\Omega\left(f_{a_{*}+\varepsilon}\right)$ are different, but their reduced nonwandering sets are the same, and their inverse limit spaces homeomorphic.

For $a>a_{\infty}, f_{a}$ has positive topological entropy, and Milnor and Thurston [25] showed that there is an entropy-preserving semiconjugacy

$$
\psi_{a}:[0,1] \rightarrow[0,1] \quad \text { such that } \psi_{a} \circ f_{a}=T_{s} \circ \psi_{a}
$$

for $\log s=h_{\text {top }}\left(f_{a}\right)$. The map $\psi_{a}$ collapses to a point every interval $K$ on which the local entropy $h_{\text {top }}\left(\left.f_{a}\right|_{K}\right)=\lim _{n} \frac{1}{n} \log l\left(\left.f_{a}^{n}\right|_{K}\right)<h_{\text {top }}\left(f_{a}\right)$. Here $l\left(\left.f_{a}^{n}\right|_{K}\right)$ is the lap-number (ie the number of maximal intervals of monotonicity) of $\left.f_{a}^{n}\right|_{K}$. This applies to wandering intervals (but logistic maps have none) and (pre)periodic intervals, with the exception of those created in the first period doubling cascade. These $2^{k}-$ periodic intervals (let us denote them by $J^{k}$ ), emerge after the critical point becomes $2^{k-1}$-periodic. For $k=1$ and 2 this happens at $a=2$ and $1+\sqrt{5}$ respectively; see Figure 8.

For $a \in\left(a_{\infty}, \tilde{a}_{k}\right]$, the semiconjugacy $\psi_{a}$ does not collapse $J^{k}$ to a point, but maps it to a $2^{k}$-periodic interval $L^{k}=\left[1-v_{k}, v_{k}\right]$. One can compute that

$$
v_{k}=\frac{1}{2}+\frac{1}{2} \prod_{j=0}^{k-1} \frac{s^{2^{j}}-1}{s^{2^{j}}+1},
$$

and $v_{k}$ or $1-v_{k}$ is the $2^{k-1}$-periodic boundary point, according to whether $k$ is odd or even. So $L^{k}$ plays the role of $J^{k}$; it exists as long as $1<s \leq 2^{1 / 2^{k}}$.

The interval $J^{k}$ continues to exist until the parameter $\tilde{a}_{k}>a_{\infty}$, where it disappears in a homoclinic bifurcation. At this parameter, $f_{\widetilde{a}_{k}}{ }^{k}(c)$ and $f_{\widetilde{a}_{k}}{ }^{2^{k+1}}(c)$ are the two boundary points of $J^{k}$ and $f_{\widetilde{a}_{k}} 2^{k+1}(c)$ is $2^{k}$-periodic. The parameters $\widetilde{a}_{k}$ form a decreasing sequence, and $\lim _{k} \tilde{a}_{k}=a_{\infty}$, due to the denseness of hyperbolicity.

Proof of Theorem 1.2 We start with the "if" direction. Recall that a logistic map can have at most one periodic attractor. Suppose that $f_{a}$ and $f_{b}$ are conjugate on their reduced nonwandering sets. If $f_{a}$ and hence $f_{b}$ have no periodic attractor (but possibly a solenoidal attractor), then denseness of hyperbolicity implies that $a=b$. In fact, in this case, the reduced nonwandering set coincides with the nonwandering set, 
and (without invoking denseness of hyperbolicity) the conjugacy $h: \Omega\left(f_{a}\right) \rightarrow \Omega\left(f_{b}\right)$ extends to a conjugacy $h:[0,1] \rightarrow[0,1]$ such that $h \circ f_{b}=f_{a} \circ h$. Hence $\underset{\lim }{\longleftarrow}\left([0,1], f_{a}\right)$ and $\lim _{\longleftarrow}\left([0,1], f_{b}\right)$ are homeomorphic.

If $f_{a}$ and hence $f_{b}$ have a periodic attractor, then they have the same period $p$ and we can choose attracting $p$-periodic points $x_{a}$ and $x_{b}$ such that spatial order $\left(f_{a}^{i}\left(x_{a}\right)\right)_{i=0}^{p-1}$ and $\left(f_{b}^{i}\left(x_{b}\right)\right)_{i=0}^{p-1}$ are the same. This means that $f_{a}$ and $f_{b}$ are in the same stage of the same period doubling cascade, and the above description implies that $\underset{\longleftarrow}{\lim }\left([0,1], f_{a}\right)$ and $\lim _{\longleftarrow}\left([0,1], f_{b}\right)$ are homeomorphic.

Now, for the "only if" direction, assume that $\lim _{\longleftarrow}\left([0,1], f_{a}\right)$ and $\lim _{\leftarrow}\left([0,1], f_{b}\right)$ are homeomorphic. We gave a complete description of the inverse limit spaces if $a<a_{\infty}$ so it suffices to consider the case $a_{\infty}<a<b$. Given a $p$-periodic interval $J$ as in (6-1), define the subcontinuum

$$
D_{J}:=\left\{x \in \underset{\lim }{\longleftarrow}\left(\left[c_{2}, c_{1}\right], f_{a}\right): x_{n p} \in J \text { for all } n \leq 0\right\} .
$$

Clearly, $D_{J}$ is $p$-periodic under the shift homeomorphism, and if $a^{\prime}$ is the parameter such that $f_{a^{\prime}}$ is conjugate to $f_{a}{ }^{p}: J \rightarrow J$, then $D_{J}$ is homeomorphic with $\lim _{\longleftarrow}\left([0,1], f_{a^{\prime}}\right)$. Also, all folding points of $\lim _{\longleftarrow}\left(\left[c_{2}, c_{1}\right], f_{a}\right)$ belong to $\bigcup_{i=0}^{p-1} \sigma^{i}\left(D_{J}\right)$, so points

$$
x \in \lim _{\longleftarrow}\left(\left[c_{2}, c_{1}\right], f_{a}\right) \backslash \bigcup_{i=0}^{p-1} \sigma^{i}\left(D_{J}\right)
$$

all have Cantor set of arcs neighborhoods in $\lim _{\leftarrow}\left(\left[c_{2}, c_{1}\right], f_{a}\right)$.

If $J$ is a maximal interval satisfying (6-1), then $D_{J}$ and its images $\sigma^{i}\left(D_{J}\right)$, for $0<i<p$, are maximal subcontinua in the sense that there is no indecomposable proper subcontinuum of the core $\lim \left(\left[c_{2}, c_{1}\right], f_{a}\right)$ that properly contains $D_{J}$ (and similarly for $\left.\sigma^{i}\left(D_{J}\right), 0<i<p\right)$. This shows that if $\underset{\leftarrow}{\lim }\left([0,1], f_{a}\right)$ and $\underset{\leftarrow}{\lim }\left([0,1], f_{b}\right)$ are homeomorphic, then their maximal periodic intervals $J_{a}$ and $J_{b}$ must have the same period, and their respective subcontinua $D_{J_{a}}$ and $D_{J_{b}}$ must be homeomorphic.

Next we will show that $J_{a}$ and $J_{b}$ are also of the same type (ie the spatial order of $\left(f_{a}^{i}\left(J_{a}\right)\right)_{i=0}^{p-1}$ and $\left(f_{b}^{i}\left(J_{b}\right)\right)_{i=0}^{p-1}$ is the same). For the factor map

$$
\begin{aligned}
& \Psi_{a}: \lim _{\longleftarrow}\left([0,1], f_{a}\right) \longrightarrow \Psi_{a}\left(\lim _{\longleftarrow}\left([0,1], f_{a}\right)\right), \\
& \left(\ldots x_{-2}, x_{-1}, x_{0}\right) \longmapsto\left(\ldots \psi_{a}\left(x_{-2}\right), \psi_{a}\left(x_{-1}\right), \psi_{a}\left(x_{0}\right)\right) \text {, }
\end{aligned}
$$

$\Psi_{a}\left(\lim _{\longleftarrow}\left([0,1], f_{a}\right)\right)$ is homeomorphic with $\lim _{\leftarrow}\left([0,1], T_{s}\right)$ for $\log s=h_{\text {top }}\left(f_{a}\right)$. Since $\psi_{a}$ collapses a periodic interval $J$ only if $\overleftarrow{\text { it }}$ does not emerge from the first period doubling cascade (ie $J \neq J^{k}$ ), we will from now on assume that $J$ is the maximal periodic interval other than (so possibly contained in some) $J^{k}$. We will show (Claims 1 
and 2, below) that $\Psi_{a}$ consists of a sequence of quotient maps that, other than squeezing a single orbit of subcontinua $\left(\sigma^{i}\left(D_{J}\right)\right)_{i=0}^{p-1}$ to $p$ endpoints, has no effect on the topology. If $J_{a}$ and $J_{b}$ are not of the same type, then $\Psi_{a}\left(\underset{\lim }{\longleftarrow}\left([0,1], f_{a}\right)\right)$ and $\Psi_{b}\left(\lim \left([0,1], f_{b}\right)\right)$ are homeomorphic with the inverse limit spaces of different tent maps, and, due to Theorem 1.1, not homeomorphic with each other. Since $\Psi_{a}$ and $\Psi_{b}$ only squeeze finitely many well-defined subcontinua to points, $\lim _{\longleftarrow}\left([0,1], f_{a}\right)$ and $\lim _{\longleftarrow}\left([0,1], f_{b}\right)$ could not have been homeomorphic.

Therefore, $J_{a}$ and $J_{b}$ are of the same type, and since the subcontinua $D_{J_{a}}$ and $D_{J_{b}}$ are homeomorphic, $\left.f_{a}\right|_{J_{a}}$ and $\left.f_{b}\right|_{J_{b}}$ must be in the same stage of their period doubling cascade, and hence their reduced nonwandering sets are conjugate. This finishes the proof, except for the announced claims:

Claim 1 Let $\Phi_{a}$ be the quotient map under the equivalence relation $x \sim y$ if $x=y$ or $x, y$ both belong to $\sigma^{i}\left(D_{J_{a}}\right)$ for the same $0 \leq i<p$. Then $\Phi_{a}\left(\lim \left([0,1], f_{a}\right)\right)$ has $p$ endpoints in the core (that are $p$-periodic under the shift) and no other folding points.

Proof Since $J_{a} \neq J^{k}$ for any of the $2^{k}$-periodic intervals emerging from the first period doubling cascade, $\partial J_{a}$ contains a $p$-periodic orientation preserving point $z_{a}$. Let

$$
\bar{z}_{a}=\left(\ldots f_{a}^{p-1}\left(z_{a}\right), z_{a}, f_{a}\left(z_{a}\right), \ldots, f_{a}^{p-1}\left(z_{a}\right)\right) ;
$$

it is a point in $D_{J_{a}}$ and one "half" of its arc-component serves as the zero-composant of $D_{J_{a}}$, while the other "half" coils densely in $\lim _{\leftarrow}\left(\left[c_{2}, c_{1}\right], f_{a}\right)$ (or densely in $D_{J^{k}}$ if $J_{a} \subset J^{k}$ for some maximal $\left.k\right)$; see [4]. The $\overleftarrow{\text { image }} \Phi_{a}\left(D_{J_{a}}\right)=\Phi_{a}\left(\bar{z}_{a}\right)$ is now an end-point of the other "half" of the arc-component of $\bar{z}_{a}$. A similar statement holds for the points $\sigma^{i}\left(\bar{z}_{a}\right), 0<i<p$. Since $\bigcup_{i=0}^{p-1} D_{J_{a}}$ contains all folding points of $\lim _{\leftarrow}\left(\left[c_{2}, c_{1}\right], f_{a}\right), \Phi_{a}\left(\lim \left(\left[c_{2}, c_{1}\right], f_{a}\right)\right)$ contains no other folding points than the $p$ -

Claim $2 \Psi_{a}\left(\lim _{\longleftarrow}\left([0,1], f_{a}\right)\right)$ is homeomorphic with $\Phi_{a}\left(\underset{\lim }{\longleftarrow}\left([0,1], f_{a}\right)\right)$.

Proof If $K$ is a maximal interval such that $f_{a}^{m}(K)=J_{a}$ for some $m \geq 0$, then there is an open neighborhood $U$ of $K$ on which $f_{a}^{m}: U \rightarrow f_{a}^{m}(U)$ is a diffeomorphism. Take $n \geq 0$ and set

$$
\bar{K}_{n}=\left\{x \in \lim _{\longleftarrow}\left([0,1], f_{a}\right): x_{-n} \in K\right\} \backslash \bigcup_{i=0}^{p-1} \sigma^{i}\left(D_{J_{a}}\right) .
$$


Since all folding points of $\lim _{\longleftarrow}\left(\left[c_{2}, c_{1}\right], f_{a}\right)$ are contained in $\bigcup_{i=0}^{p-1} \sigma^{i}\left(D_{J_{a}}\right), \bar{K}_{n}$ is a zero-dimensional set of arcs (more precisely, this zero-dimensional set is a Cantor set together with a countable set coming from the zero-composant). Similarly

$$
\bar{U}_{n}=\left\{x \in \lim _{\longleftarrow}\left([0,1], f_{a}\right): x_{-n} \in U\right\} \backslash \bigcup_{i=0}^{p-1} \sigma^{i}\left(D_{J_{a}}\right)
$$

is a zero-dimensional set of open arcs, and $\bar{U}_{n}$ compactly contains $\bar{K}_{n}$. Set $x \sim \bar{K}_{n} y$ if $x=y$ or if $x$ and $y$ both belong to $\bar{K}_{n}$. It follows that $\lim \left([0,1], f_{a}\right)$ is homeomorphic with $\lim _{\longleftarrow}\left([0,1], f_{a}\right) / \sim_{\bar{K}_{n}}$ for each component $K$ of $\overleftarrow{U}_{m \geq 0} f_{a}^{-m}\left(J_{a}\right)$ and $n \geq 0$.

The collection

$$
\mathcal{K}:=\left\{\bar{K}_{n}: K \text { is component of } \bigcup_{m \geq 0} f_{a}^{-m}\left(J_{a}\right), n \geq 0\right\}
$$

is a null-sequence, ie for fixed $\delta>0$, there are only finitely many elements in $\mathcal{K}$ with $\operatorname{diam}\left(\bar{K}_{n}\right)>\delta$. Therefore, if we denote by $\sim \mathcal{K}$ the intersection of all equivalence relations $\sim \bar{K}_{n}, \bar{K}_{n} \in \mathcal{K}, \Phi_{a}\left(\lim _{\leftarrow}\left([0,1], f_{a}\right)\right)$ is homeomorphic with $\Phi_{a}\left(\lim _{\longleftarrow}\left([0,1], f_{a}\right)\right) / \sim_{\mathcal{K}}$ which in turn is homeomorphic with $\Psi_{a}\left(\lim _{\longleftarrow}\left([0,1], f_{a}\right)\right)$

This proves the claims and hence Theorem 1.2.

Acknowledgement Part of this research was done at the Mathematisches Forschungsinstitut Oberwolfach during a stay within the Research in Pairs Programme from January 11 to January 24, 2009. The authors thank the MFO for its hospitality. Henk Bruin also thanks Delft University of Technology, where this paper was largely completed. Sonja Štimac is supported in part by the NSF grant number 0604958 and in part by the MZOS grant number 037-0372791-2802 of the Republic of Croatia.

\section{References}

[1] J E Anderson, I F Putnam, Topological invariants for substitution tilings and their associated $C^{*}$-algebras, Ergodic Theory Dynam. Systems 18 (1998) 509-537 MR1631708

[2] M Barge, K M Brucks, B Diamond, Self-similarity in inverse limit spaces of the tent family, Proc. Amer. Math. Soc. 124 (1996) 3563-3570 MR1363409

[3] M Barge, B Diamond, Homeomorphisms of inverse limit spaces of one-dimensional maps, Fund. Math. 146 (1995) 171-187 MR1314982

[4] M Barge, B Diamond, Inverse limit spaces of infinitely renormalizable maps, Topology Appl. 83 (1998) 103-108 MR1604798 
[5] M Barge, B Diamond, Subcontinua of the closure of the unstable manifold at a homoclinic tangency, Ergodic Theory Dynam. Systems 19 (1999) 289-307 MR1685395

[6] M Barge, S Holte, Nearly one-dimensional Hénon attractors and inverse limits, Nonlinearity 8 (1995) 29-42 MR1313141

[7] M Barge, W T Ingram, Inverse limits on [0, 1] using logistic bonding maps, Topology Appl. 72 (1996) 159-172 MR1404274

[8] L Block, S Jakimovik, L Kailhofer, J Keesling, On the classification of inverse limits of tent maps, Fund. Math. 187 (2005) 171-192 MR2214878

[9] L Block, S Jakimovik, J Keesling, On Ingram's conjecture, Topology Proc. 30 (2006) 95-114 MR2280661

[10] L Block, J Keesling, B E Raines, S Štimac, Homeomorphisms of unimodal inverse limit spaces with a non-recurrent critical point, Topology Appl. 156 (2009) 2417-2425 MR2546944

[11] K M Brucks, H Bruin, Subcontinua of inverse limit spaces of unimodal maps, Fund. Math. 160 (1999) 219-246 MR1708988

[12] K M Brucks, B Diamond, Monotonicity of auto-expansions, Phys. D 51 (1991) 39-42 MR1128801

[13] H Bruin, Planar embeddings of inverse limit spaces of unimodal maps, Topology Appl. 96 (1999) 191-208 MR1709688

[14] H Bruin, Inverse limit spaces of post-critically finite tent maps, Fund. Math. 165 (2000) 125-138 MR1808727

[15] H Bruin, Subcontinua of Fibonacci-like inverse limit spaces, Topology Proc. 31 (2007) 37-50 MR2363150

[16] A Douady, Topological entropy of unimodal maps: monotonicity for quadratic polynomials, from: "Real and complex dynamical systems", (B Branner, P Hjorth, editors), NATO Adv. Sci. Inst. Ser. C Math. Phys. Sci. 464, Kluwer Acad. Publ., Dordrecht (1995) 65-87

[17] M J Feigenbaum, Quantitative universality for a class of nonlinear transformations, J. Statist. Phys. 19 (1978) 25-52 MR0501179

[18] J Graczyk, G Świa̧tek, Generic hyperbolicity in the logistic family, Ann. of Math. 146 (1997) 1-52 MR1469316

[19] J Guckenheimer, Sensitive dependence to initial conditions for one-dimensional maps, Comm. Math. Phys. 70 (1979) 133-160 MR553966

[20] F Hofbauer, P Raith, K Simon, Hausdorff dimension for some hyperbolic attractors with overlaps and without finite Markov partition, Ergodic Theory Dynam. Systems 27 (2007) 1143-1165 MR2342970 
[21] W T Ingram, Inverse limits on [0,1] using tent maps and certain other piecewise linear bonding maps, from: "Continua", (H Cook, W T Ingram, K T Kuperberg, A Lelek, P Minc, editors), Lecture Notes in Pure and Appl. Math. 170, Dekker, New York (1995) 253-258 MR1326847

[22] L Kailhofer, A classification of inverse limit spaces of tent maps with periodic critical points, Fund. Math. 177 (2003) 95-120 MR1992527

[23] M Lyubich, Dynamics of quadratic polynomials. I, II, Acta Math. 178 (1997) 185-247, 247-297 MR1459261

[24] W de Melo, S van Strien, One-dimensional dynamics, Ergeb. Math. Grenzgeb. 25, Springer, Berlin (1993) MR1239171

[25] J Milnor, W Thurston, On iterated maps of the interval, from: "Dynamical systems", (J C Alexander, editor), Lecture Notes in Math. 1342, Springer, Berlin (1988) 465-563 MR970571

[26] M Misiurewicz, Embedding inverse limits of interval maps as attractors, Fund. Math. 125 (1985) 23-40 MR813986

[27] M Misiurewicz, W Szlenk, Entropy of piecewise monotone mappings, Studia Math. 67 (1980) 45-63 MR579440

[28] B E Raines, Inhomogeneities in non-hyperbolic one-dimensional invariant sets, Fund. Math. 182 (2004) 241-268 MR2098780

[29] B E Raines, S Štimac, A classification of inverse limit spaces of tent maps with a nonrecurrent critical point, Algebr. Geom. Topol. 9 (2009) 1049-1088 MR2511139

[30] D Singer, Stable orbits and bifurcation of maps of the interval, SIAM J. Appl. Math. 35 (1978) 260-267 MR0494306

[31] S Štimac, A classification of inverse limit spaces of tent maps with finite critical orbit, Topology Appl. 154 (2007) 2265-2281 MR2328011

[32] R Swanson, $\mathbf{H}$ Volkmer, Invariants of weak equivalence in primitive matrices, Ergodic Theory Dynam. Systems 20 (2000) 611-626 MR1756989

[33] W Szczechla, Inverse limits of certain interval mappings as attractors in two dimensions, Fund. Math. 133 (1989) 1-23 MR1059158

[34] C Tresser, $\mathbf{P}$ Coullet, Itérations d'endomorphismes et groupe de renormalisation, C. R. Acad. Sci. Paris Sér. A-B 287 (1978) A577-A580 MR512110

[35] R F Williams, One-dimensional non-wandering sets, Topology 6 (1967) 473-487 MR0217808

[36] R F Williams, Classification of one dimensional attractors, from: "Global Analysis", Proc. Symp. Pure Math. 14, Amer. Math. Soc. (1970) 341-361 MR0266227 
Department of Mathematical Sciences, Montana State University Bozeman, MT 59717, USA

Faculty of Mathematics, University of Vienna

Nordbergstrasse 15, 1090 Wien, Austria

Department of Mathematics, University of Zagreb

Bijenička 30, 10000 Zagreb, Croatia

umsfmbar@math.montana.edu, henk.bruin@univie.ac.at, sonja@math.hr http://www . math.montana.edu/ umsfmbar/, http://www.mat.univie.ac.at/ bruin/, http://www.math.hr/ sonja

Proposed: David Gabai

Seconded: Leonid Polterovich, Steve Ferry

Received: 7 June 2012 Accepted: 14 August 2012 